ESAIM: COCV 19 (2013) 129-166

DOI: $10.1051 / \mathrm{cocv} / 2012002$
ESAIM: Control, Optimisation and Calculus of Variations

www.esaim-cocv.org

\title{
A HAMILTON-JACOBI APPROACH TO JUNCTION PROBLEMS AND APPLICATION TO TRAFFIC FLOWS*
}

\author{
Cyril Imbert ${ }^{1,2}$, RÉGis Monneau $^{3}$ and Hasnaa Zidani ${ }^{4}$
}

\begin{abstract}
This paper is concerned with the study of a model case of first order Hamilton-Jacobi equations posed on a "junction", that is to say the union of a finite number of half-lines with a unique common point. The main result is a comparison principle. We also prove existence and stability of solutions. The two challenging difficulties are the singular geometry of the domain and the discontinuity of the Hamiltonian. As far as discontinuous Hamiltonians are concerned, these results seem to be new. They are applied to the study of some models arising in traffic flows. The techniques developed in the present article provide new powerful tools for the analysis of such problems.
\end{abstract}

Mathematics Subject Classification. 35F21, 35D40, 35Q93, 35R05, 35B51.

Received July 12, 2011.

Published online 1st March 2012.

\section{INTRODUCTION}

In this paper we are interested in Hamilton-Jacobi (HJ) equations posed on a one dimensional domain containing one single singularity. This is a special case of a more general setting where HJ equations are posed in domains that are unions of submanifolds whose dimensions are different [8]. An intermediate setting is the study of HJ equations on networks, see in particular [1]. We will restrict ourselves to a very simple network: the union of a finite numbers of half-lines of the plane with a single common point. Such a domain is referred to as a junction and the common point is called the junction point. We point out that getting a comparison principle is the most difficult part in such a study; it is obtained in [1] for similar special networks (bounded star-shaped ones). Our motivation comes from traffic flows. For this reason, it is natural to impose different dynamics on each branch of the junction. Consequently, the resulting Hamiltonian is by nature discontinuous

Keywords and phrases. Hamilton-Jacobi equations, discontinuous Hamiltonians, viscosity solutions, optimal control, traffic problems, junctions.

* Dedicated to J.-B. Hiriart-Urruty.

1 Université Paris-Dauphine, CEREMADE, UMR CNRS 7534, place de Lattre de Tassigny, 75775 Paris Cedex 16, France. imbert@ceremade.dauphine.fr

2 École Normale Supérieure, Département de Mathématiques et Applications, UMR 8553, 45 rue d’Ulm, 75230 Paris Cedex 5, France

3 Université Paris-Est, École des Ponts ParisTech, CERMICS, 6 et 8 avenue Blaise Pascal, Cité Descartes Champs-sur-Marne, 77455 Marne-La-Vallée Cedex 2, France. monneau@cermics.enpc.fr

4 ENSTA ParisTech \& INRIA Saclay (Commands INRIA team), 32 boulevard Victor, 75379 Paris Cedex 15, France. Hasnaa.Zidani@ensta.fr 


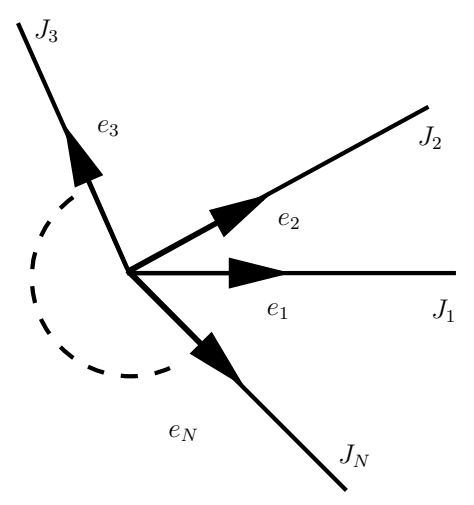

Figure 1. A junction.

at the junction point. Together with the singularity of the domain, this is the major technical difficulty to overcome. The analysis relies on the complete study of some minimal action (or metric) related to the optimal control interpretation of the equation $[16,33]$. We prove in particular that this minimal action is semi-concave by computing it.

We first present the problem and the main results in details. Then we recall existing results and compare them with ours.

\subsection{Setting of the problem}

In this subsection, the analytical problem is introduced in details. We first define the junction, then the space of functions on the junction and finally the Hamilton-Jacobi equation.

The junction. Let us consider $N \geq 1$ different unit vectors $e_{i} \in \mathbb{R}^{2}$ for $i=1, \ldots, N$. We define the branches

$$
J_{i}=[0,+\infty) \cdot e_{i}, \quad J_{i}^{*}=J_{i} \backslash\{0\}, \quad i=1, \ldots, N
$$

and the junction (see Fig. 1)

$$
J=\bigcup_{i=1, \ldots, N} J_{i}
$$

The origin $x=0$ is called the junction point. It is also useful to write $J^{*}=J \backslash\{0\}$. For a time $T>0$, we also define

$$
J_{T}=(0, T) \times J .
$$

The reader can remark that we chose to embed the junction in a two-dimensional Euclidean space. But we could also have considered an abstract junction, or we could have embedded it for instance in a higher dimensional Euclidean space. We made such a choice for the sake of clarity.

Space of functions. For a function $u: J_{T} \rightarrow \mathbb{R}$, we denote by $u^{i}$ the restriction of $u$ to $(0, T) \times J_{i}$. Then we define the natural space of functions on the junction

$$
C_{*}^{1}\left(J_{T}\right)=\left\{u \in C\left(J_{T}\right), \quad u^{i} \in C^{1}\left((0, T) \times J_{i}\right) \quad \text { for } \quad i=1, \ldots, N\right\} .
$$

In particular for $u \in C_{*}^{1}\left(J_{T}\right)$ and $x=x_{i} e_{i}$ with $x_{i} \geq 0$, we define

$$
u_{t}(t, x)=\frac{\partial u^{i}}{\partial t}\left(t, x_{i} e_{i}\right) \quad \text { and } \quad u_{x}^{i}(t, x)=\frac{\partial u^{i}}{\partial x_{i}}\left(t, x_{i} e_{i}\right) .
$$

Then we set

$$
\begin{cases}u_{x}(t, x)=u_{x}^{i}(t, x) & \text { if } \quad x \neq 0 \\ u_{x}(t, 0)=\left(u_{x}^{j}(t, 0)\right)_{j=1, \ldots, N} & \text { if } \quad x=0 .\end{cases}
$$


HJ equation on the junction. We are interested in continuous functions $u:[0, T) \times J \rightarrow \mathbb{R}$ which are viscosity solutions (see Def. 3.2) on $J_{T}$ of

$$
u_{t}+H\left(x, u_{x}\right)=0
$$

submitted to an initial condition

$$
u(0, x)=u_{0}(x), \quad x \in J .
$$

Because of the optimal control problem we have in mind (see Sect. 1.1 below), we restrict ourselves to the simplest case of discontinuous Hamiltonians; precisely, we consider

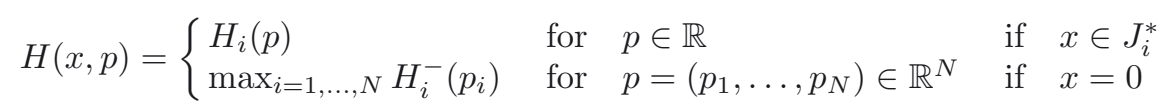

where $H_{i}$ are convex functions whose Legendre-Fenchel transform is denoted by $L_{i}$. We recall that

$$
H_{i}(p)=L_{i}^{*}(p)=\sup _{q \in \mathbb{R}}\left(p q-L_{i}(q)\right)
$$

and

$$
H_{i}=L_{i}^{*}
$$

We also consider

$$
H_{i}^{-}\left(p_{i}\right)=\sup _{q \leq 0}\left(p_{i} q-L_{i}(q)\right) .
$$

Therefore equation (1.1) can be rewritten as follows

$$
\begin{cases}u_{t}^{i}+H_{i}\left(u_{x}^{i}\right)=0 & \text { on } \quad(0, T) \times J_{i}^{*} \\ u_{t}+\max _{i=1, \ldots, N} H_{i}^{-}\left(u_{x}^{i}\right)=0 & \text { on } \quad(0, T) \times\{0\} .\end{cases}
$$

The optimal control framework. In this paragraph, we give an optimal control interpretation $[4,7,26]$ of the Hamilton-Jacobi equation. We define the set of admissible controls at a point $x \in J$ by

$$
A(x)= \begin{cases}\mathbb{R} e_{i_{0}} & \text { if } \quad x \in J_{i_{0}}^{*} \\ \bigcup_{i=1, \ldots, N} \mathbb{R}^{+} e_{i} & \text { if } \quad x=0\end{cases}
$$

For $(s, y),(t, x) \in[0, T] \times J$ with $s \leq t$ (the case $s=t$ being trivial and forcing $y=x$ ), we define the set of admissible trajectories from $(s, y)$ to $(t, x)$ by

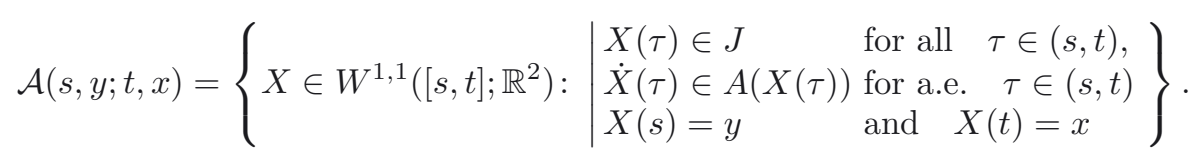

For $P=p e_{i} \in A(x)$ with $p \in \mathbb{R}$, we define the Lagrangian on the junction

$$
L(x, P)=\left\{\begin{array}{lll}
L_{i}(p) & \text { if } \quad & x \in J_{i}^{*} \\
L_{0}(p) & \text { if } \quad x=0
\end{array}\right.
$$

with

$$
L_{0}(p)=\min _{j=1, \ldots, N} L_{j}(p)
$$

The reader can be surprised by the definition of $L(x, P)$ for $x=0$. In fact, if one considers only trajectories that do not stay at the junction point, then the ones staying at 0 are approximated by those staying very close to 0 on a branch $i \in I_{0}$ and moving "slowly" $(\dot{X} \simeq 0)$. 


\subsection{Main results}

We make the following assumptions:

(A0) The initial data $u_{0}$ is Lipschitz continuous.

(A1) There exists a constant $\gamma>0$, and for all $i=1, \ldots, N$, there exists $C^{2}(\mathbb{R})$ functions $L_{i}$ satisfying $L_{i}^{\prime \prime} \geq \gamma>0$, such that (1.3) and (1.4) hold.

Theorem 1.1 (existence and uniqueness). Assume (A0)-(A1) and let $T>0$. Then there exists a unique viscosity solution $u$ of (1.1)-(1.2) on $J_{T}$ in the sense of Definition 3.2, satisfying for some constant $C_{T}>0$

$$
\left|u(t, x)-u_{0}(x)\right| \leq C_{T} \quad \text { for all } \quad(t, x) \in J_{T} .
$$

Moreover the function $u$ is Lipschitz continuous with respect to $(t, x)$ on $J_{T}$.

On one hand, we will see below that the existence of a solution can be obtained with Perron's method under weaker assumptions than (A1) (see Thm. A.2). On the other hand, we are able to get uniqueness of the solution only under assumption (A1) and this is a consequence of the following result:

Theorem 1.2 (comparison principle). Assume (A0)-(A1). Let $T>0$ and let $u$ (resp. $v$ ) be a sub-solution (resp. a super-solution) of (1.1)-(1.2) on $J_{T}$ in the sense of Definition 3.2. We also assume that there exists a constant $C_{T}>0$ such that for all $(t, x) \in J_{T}$

$$
u(t, x) \leq C_{T}(1+|x|) \quad\left(\text { resp. } \quad v(t, x) \geq-C_{T}(1+|x|)\right) .
$$

Then we have $u \leq v$ on $J_{T}$.

In order to prove this strong uniqueness result, we will use in an essential way the value function associated to the optimal control problem described in Section 1.1: for $t \geq 0$,

$$
u_{\mathrm{oc}}(t, x)=\inf _{y \in J, X \in \mathcal{A}(0, y ; t, x)}\left\{u_{0}(y)+\int_{0}^{t} L(X(\tau), \dot{X}(\tau)) \mathrm{d} \tau\right\}
$$

where $L$ is defined in (1.7) and $\mathcal{A}(0, y ; t, x)$ is defined in (1.6).

Theorem 1.3 (optimal control representation of the solution). Assume (A0)-(A1) and let $T>0$. The unique solution given by Theorem 1.1 is $u=u_{\mathrm{oc}}$ with $u_{\mathrm{oc}}$ given in (1.8). Moreover, we have the following Hopf-Lax representation formula

$$
u_{\mathrm{oc}}(t, x)=\inf _{y \in J}\left\{u_{0}(y)+\mathcal{D}(0, y ; t, x)\right\}
$$

with

$$
\mathcal{D}(0, y ; t, x)=\inf _{X \in \mathcal{A}(0, y ; t, x)}\left\{\int_{0}^{t} L(X(\tau), \dot{X}(\tau)) \mathrm{d} \tau\right\} .
$$

The comparison principle is obtained by combining

- a super-optimality principle for super-solutions $v$, which implies $v \geq u_{\mathrm{oc}}$;

- a direct comparison result with sub-solutions $u$, which gives $u_{\mathrm{oc}} \geq u$.

We finally have the following result which shed light on the role of the junction condition (see the second line of $(1.5))$.

Theorem 1.4 (comparison with continuous solutions outside the junction point). Assume (A0)-(A1) and let $T>0$. Let $u \in C([0, T) \times J)$ be such that $u(0, \cdot)=u_{0}$ and for each $i \in\{1, \ldots, N\}$, the restriction $u^{i}$ of $u$ to $(0, T) \times J_{i}$ is a classical viscosity solution of

$$
u_{t}^{i}+H_{i}\left(u_{x}^{i}\right)=0 \quad \text { on } \quad(0, T) \times J_{i}^{*} .
$$

Then $u$ is a sub-solution of (1.1)-(1.2) on $J_{T}$ in the sense of Definition 3.2, and $u \leq u_{\mathrm{oc}}$. 
An immediate consequence of Theorem 1.4 is the fact that the optimal control solution $u_{\mathrm{oc}}$ is the maximal continuous function which is a viscosity solution on each open branch.

We apply in Section 2 our HJ approach to describe traffic flows on a junction. In particular, we recover the well-known junction conditions of Lebacque (see [22]) or equivalently those for the Riemann solver at the junction as in the book of Garavello and Piccoli [17]; see also [18].

\subsection{Comments}

We already mentioned that the main difficulties we have to overcome in order to get our main results are on one hand the singular geometry of the domain and on the other hand the discontinuity of the Hamiltonian.

Discontinuity. Several papers in the literature deal with HJB equations with discontinuous coefficients; see for instance $[6,9,12,13,28,34,37-39]$. Note that in these works the optimal trajectories do cross the regions of discontinuities (i.e. the junction in the present paper) only on a set of time of measure zero. In the present paper, the optimal trajectories can remain on the junction during some time intervals, and the results cited above do not apply then to the problem studied here.

On the other hand, the analysis of scalar conservation laws with discontinuous flux functions has been extensively studied, we refer to $[3,10,32]$ and references therein. We also point out that a uniqueness result is proved in $\mathbb{R}$ in the framework corresponding a junction with two branches [21]. To the best of our knowledge, in the case of junctions with more than two branches, there are no uniqueness result. Moreover, the link between HJB equations and conservation laws with discontinuous has been seldom investigated [29].

The main differences between the study in [1] and the one carried out in the present paper lie in the fact that in [1] the Lagrangian can depend on $x$ and is continuous with respect to this variable, while we consider a Lagrangian which is constant in $x$ on each branch but can be discontinuous (with respect to $x$ ) at the junction. We point out that we cannot extend directly our approach to Lagrangians depending on $x$ since we use extensively the representation formula "à la Hopf-Lax". In order to generalize results in this direction, the semi-concavity of the "fundamental solution" $\mathcal{D}$ should be proved without relying on explicit computations. This question is very interesting but is out of the scope of the present paper.

Networks. It is by now well known that the study of traffic flows on networks is an important motivation that give rise to several difficulties related to scalar conservation laws with discontinuous coefficients. This topic has been widely studied by many authors, see for instance $[10,15,17]$ and the references therein.

However, the study of HJB equations on networks has been considered very recently; the reader is referred to $[11,31]$ where Eikonal equations are considered. A more general framework was also studied in $[1,2]$ where a definition of viscosity solutions on networks, similar to Definition 3.2, has been introduced.

It would be interesting to extend the results of the present paper to more general networks but the obstacle is the same than the one to be overcome if one wants to deal with Lagrangians depending on $x$ : for a general network, the complete study of the fundamental solution is probably out of reach. This is the reason why we only consider the very specific case of a junction in order to be able to overcome the difficulty of the discontinuity of the Lagrangian.

The optimal control interpretation. As explained above, the comparison principle is proved by using in an essential way the optimal control interpretation of the Hamilton-Jacobi equation. The use of representation formulas and/or optimality principles is classical in the study of Hamilton-Jacobi equations [19, 20, 27, 35, 36]. More specifically, it is also known that a "metric" interpretation of the Hamilton-Jacobi equation is fruitful [33]. Such an interpretation plays a central role in the weak KAM theory [16].

As far as our problem is concerned, we are not able to prove uniqueness of viscosity solutions by using the classical techniques of doubling variable. The idea used here is based on the equivalence between the viscosity super-solution and the super-optimality principle (also known as weak-invariance principle), and by using representation formulas for the viscosity sub-solutions. This representation seems to be new for HJB equations with discontinuous coefficients, see for instance [14]. 
We would like next to be a bit more precise. The technical core of the paper lies in Theorem 4.2. This result implies that the function

$$
\mathcal{D}(s, y ; t, x)=(t-s) \mathcal{D}_{0}\left(\frac{y}{t-s}, \frac{x}{t-s}\right)
$$

is semi-concave with respect to $(t, x)$ and $(s, y)$ and, if there are at least two branches $(N \geq 2)$, that $\mathcal{D}$ satisfies

$$
\left\{\begin{aligned}
\mathcal{D}_{t}+H\left(x, \mathcal{D}_{x}\right) & =0 \\
-\mathcal{D}_{s}+H\left(y,-\mathcal{D}_{y}\right) & =0
\end{aligned}\right.
$$

(in a weak sense made precise in the statement of Thm. 4.2). In the case where the Lagrangians coincide at the junction point $\left(L_{1}(0)=\ldots=L_{N}(0)\right)$, it turns out that the restriction $\mathcal{D}_{0}^{j i}(y, x)$ of $\mathcal{D}_{0}$ to $J_{j} \times J_{i}$ belongs to $C^{1}\left(J_{j} \times J_{i}\right)$ and is convex. A more general case is considered in this paper: Lagrangians can differ at the junction point and in this case, the functions $\mathcal{D}_{0}^{j i}$ are not convex nor $C^{1}$ anymore for some $(i, j)$. Let us point out here that the assumptions on the Hamiltonian $H_{i}$, and in particular the fact that it does not depend on the space variable $x$, plays a crucial role to establish the properties satisfied by the minimal action function $\mathcal{D}$.

Generalization and open problems. Eventually, we briefly mention natural generalizations of our results and some important open problems. First of all, it would be natural to extend the results of this paper to more general setting where the Hamiltonians $H_{i}$ depend on the space variable $x$. Moreover, it would be interesting to consider general networks with several junction points. Dealing with non-convex and non-coercive Hamiltonians is quite challenging and would require first to have a direct proof of the comparison principle which does not need to go through the interpretation of the viscosity solution as the value function of an optimal control problem.

\section{Organization of the article}

Section 2 is devoted to the application of our results to some traffic flow problems. In particular, the HJ equation is derived and the junction condition is interpreted. In Section 3, the definition of (viscosity) solutions is made precise. In Section 4, the first important properties of optimal trajectories are given. Section 5 is devoted to the proof of the main results of the paper. In particular, the comparison principle is derived by proving a super-optimality principle and by comparing sub-solutions with the solution given by the optimal control interpretation of the equation. Section 6 is devoted to the proof of the technical core of the paper, namely the existence of test functions for the minimal action associated with the optimal control interpretation.

\section{Notation}

Distance and coordinates in the junction. We denote by $d$ the geodesic distance defined on $J$ by

$$
d(x, y)=\left\{\begin{array}{llll}
|x-y| & \text { if } & x, y & \text { belong to the same branch } J_{i} \text { for some } i \\
|x|+|y| & \text { if } & x, y & \text { do not belong to the same branch. }
\end{array}\right.
$$

For $x \in J, B(x, r)$ denotes the (open) ball centered at $x$ of radius $r$. We also consider balls $B((t, x), r)$ centered at $(t, x) \in(0,+\infty) \times J$ of radius $r>0$. For $x \in J$, let us define the index $i(x)$ of the branch where $x$ lies. Precisely we set:

$$
i(x)=\left\{\begin{array}{lll}
i_{0} & \text { if } & x \in J_{i_{0}}^{*} \\
0 & \text { if } & x=0
\end{array}\right.
$$

Up to reordering the indices, we assume that there exists an index $k_{0} \in\{1, \ldots, N\}$ such that

$$
L_{0}(0)=L_{1}(0)=\cdots=L_{k_{0}}(0)<L_{k_{0}+1}(0) \leq \cdots \leq L_{N}(0) .
$$

We also set

$$
I_{0}=\left\{1, \ldots, k_{0}\right\} \quad \text { and } \quad I_{N}=\{1, \ldots, N\}
$$




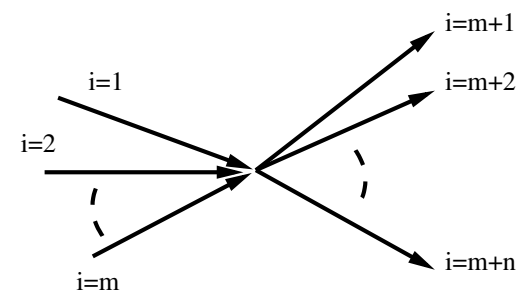

Figure 2. A traffic junction.

Functions defined in $\mathbf{J}^{2}$. For a function $\varphi$ defined on $J \times J$, we call $\varphi^{i j}$ its restriction to $J_{i} \times J_{j}$. Then we define the space

$$
C_{*}^{1}\left(J^{2}\right)=\left\{\varphi \in C\left(J^{2}\right), \quad \varphi^{i j} \in C^{1}\left(J_{i} \times J_{j}\right) \quad \text { for all } i, j \in I_{N}\right\} .
$$

We also call for $x=x_{i} e_{i}$ with $x_{i} \geq 0$ and $y=y_{j} e_{j}$ with $y_{j} \geq 0$

$$
\partial_{x}^{i} \varphi(x, y)=\frac{\partial}{\partial x_{i}} \varphi^{i j}\left(x_{i} e_{i}, y\right) \quad \text { and } \quad \partial_{y}^{j} \varphi(x, y)=\frac{\partial}{\partial y_{j}} \varphi^{i j}\left(x, y_{j} e_{j}\right)
$$

and

$$
\partial_{x} \varphi(x, y)= \begin{cases}\partial_{x}^{i} \varphi(x, y) & \text { if } \quad x \in J_{i}^{*} \\ \left(\partial_{x}^{i} \varphi(x, y)\right)_{i=1, \ldots, N} & \text { if } \quad x=0\end{cases}
$$

and similarly

$$
\partial_{y} \varphi(x, y)= \begin{cases}\partial_{y}^{j} \varphi(x, y) & \text { if } \quad y \in J_{j}^{*} \\ \left(\partial_{y}^{j} \varphi(x, y)\right)_{j=1, \ldots, N} & \text { if } \quad y=0\end{cases}
$$

We also set

$$
\begin{cases}x \partial_{x} \varphi(x, y)=x_{i} \partial_{x}^{i} \varphi(x, y) & \text { if } \quad x \in J_{i}, \\ y \partial_{y} \varphi(x, y)=y_{j} \partial_{y}^{j} \varphi(x, y) & \text { if } \quad y \in J_{j} .\end{cases}
$$

\section{Applichtion to the modeling of traffic flows}

In this section we present the derivation of the Hamilton-Jacobi formulation of traffic on a junction. We also discuss the meaning of our junction condition in this framework and relate it to known results.

\subsection{Primitive of the densities of cars}

We consider a junction (represented in Fig. 2) with $m \geq 1$ incoming roads (labeled by the index $i=1, \ldots, m$ ) and $n \geq 1$ outgoing roads (labeled by $j=m+1, \ldots, m+n=N$ ). This means that the cars move on the incoming roads in the direction of the junction and then have to choose to go on one of the $n$ outgoing roads. We assume that the proportion of cars coming from the branch $i=1, \ldots, m$ is a fixed number $\gamma_{i}>0$ (which may be not realistic for $m \geq 2$ ), and that the proportion of cars going on each branch $j \in\{m+1, \ldots, m+n\}$ is also a fixed number $\gamma_{j}>0$. We also assume the obvious relations (for conservation of cars)

$$
\sum_{i=1, \ldots, m} \gamma_{i}=1 \text { and } \sum_{j=m+1, \ldots, m+n} \gamma_{j}=1
$$

We denote by $\rho^{k}(t, X) \geq 0$ the car density at time $t$ and at the position $X$ on the branch $k$. In particular, we assume that the traffic is described on each branch $k$ by a flux function $f^{k}: \mathbb{R} \rightarrow \mathbb{R}$. We assume

each function $f^{k}$ is concave and has a unique maximum value at $\rho=\rho_{c}^{k}>0$. 
The typical example of such flux function is given by the LWR model (Lighthill and Whitham [25] and Richards [30]), with

$$
f(\rho)=\rho v(\rho) \quad \text { with the velocity } \quad v(\rho)=V_{\max }\left(1-\rho / \rho_{\max }\right)
$$

where $V_{\max }$ and $\rho_{\max }$ are respectively the maximal velocity and the maximal car density in the model). In this model the critical car density $\rho_{c}$ where $f$ is maximal, is equal to $\frac{1}{2} \rho_{\max }$.

We assume that the car densities are solution of non linear transport equations:

$$
\begin{cases}\rho_{t}^{i}+\left(f^{i}\left(\rho^{i}\right)\right)_{X}=0, \quad X<0, & \text { for } \quad i=1, \ldots, m \\ \rho_{t}^{j}+\left(f^{j}\left(\rho^{j}\right)\right)_{X}=0, \quad X>0, & \text { for } \quad j=m+1, \ldots, m+n\end{cases}
$$

where we assume that the junction point is located at the origin $X=0$.

We do not precise yet the junction condition at $X=0$, and we now proceed formally to deduce the HamiltonJacobi model of such a junction. For a function $g$, that will be defined precisely later, let us consider the functions

$$
\left\{\begin{array}{lll}
U^{i}(t, X)=g(t)+\frac{1}{\gamma^{i}} \int_{0}^{X} \rho^{i}(t, Y) \mathrm{d} Y, & X<0, & \text { for } \quad i=1, \ldots, m, \\
U^{j}(t, X)=g(t)+\frac{1}{\gamma^{j}} \int_{0}^{X} \rho^{j}(t, Y) \mathrm{d} Y, & X>0, & \text { for } \quad j=m+1, \ldots, m+n .
\end{array}\right.
$$

Then we can compute formally for $j=m+1, \ldots, m+n$

$$
\begin{aligned}
U_{t}^{j} & =g^{\prime}(t)+\frac{1}{\gamma^{j}} \int_{0}^{X} \rho_{t}^{j}(t, Y) \mathrm{d} Y \\
& =g^{\prime}(t)-\frac{1}{\gamma^{j}} \int_{0}^{X}\left(f^{j}\left(\rho^{j}(t, Y)\right)\right)_{X} \mathrm{~d} Y \\
& =-\frac{1}{\gamma^{j}} f^{j}\left(\rho^{j}(t, X)\right)+g^{\prime}(t)+\frac{1}{\gamma^{j}} f^{j}\left(\rho^{j}\left(t, 0^{+}\right)\right) .
\end{aligned}
$$

This shows that for $j=m+1, \ldots, m+n$

$$
U_{t}^{j}+\frac{1}{\gamma^{j}} f^{j}\left(\gamma_{j} U_{X}^{j}\right)=h^{j}(t)
$$

where

$$
h^{j}(t):=g^{\prime}(t)+\frac{1}{\gamma^{j}} f^{j}\left(\rho^{j}\left(t, 0^{+}\right)\right)
$$

Remark that we can show similarly that $(2.5)$ is still true for the index $j$ replaced by $i=1, \ldots, m$ with

$$
h^{i}(t)=g^{\prime}(t)+\frac{1}{\gamma^{i}} f^{i}\left(\rho^{i}\left(t, 0^{-}\right)\right) .
$$

In particular, this shows (at least when the quantities in (2.5) are well defined) that we can choose $g$ such that the total flux $-g^{\prime}(t)$ is given by

$$
-g^{\prime}(t)=\sum_{i=1, \ldots, m} f^{i}\left(\rho^{i}\left(t, 0^{-}\right)\right)
$$

and then we have

$$
h^{k}(t) \equiv 0 \quad \text { for } \quad i=1, \ldots, m+n
$$

if and only if

$$
\left\{\begin{array}{l}
f^{i}\left(\rho^{i}(t, 0-)\right)=\gamma^{i}\left(-g^{\prime}(t)\right) \quad \text { for } \quad i=1, \ldots, m \\
f^{j}\left(\rho^{j}\left(t, 0^{+}\right)\right)=\gamma^{j}\left(-g^{\prime}(t)\right) \quad \text { for } \quad j=m+1, \ldots, m+n
\end{array}\right.
$$


which is exactly the expected condition which says that the proportion of incoming cars going in the junction from the branch $i$ is $\gamma^{i}$ and the proportion of cars getting out of the junction which choose to go on the branch $j$ is $\gamma^{j}$.

Let us notice that if we choose the initial condition $g(0)=0$, then we deduce from (2.6) that we have for $l=1, \ldots, m+n$

$$
-U^{l}(t, 0)=-g(t)=\int_{0}^{t} \sum_{i=1, \ldots, m} f^{i}\left(\rho^{i}\left(\tau, 0^{-}\right)\right) \mathrm{d} \tau
$$

which shows that $-U^{l}(t, 0)$ can be interpreted as the total quantity of cars passing through the junction point $X=0$ during the time interval $[0, t)$. As a consequence, the quantity $-U_{t}^{l}(t, 0)$ can also be interpreted as the instantaneous flux of cars passing through the junction point.

We now give a further interpretation of the problem in the special case $m=1$. In the special case $m=1$, imagine for a moment, that we come back to a discrete description of the traffic, where each car of label $k$ has a position $x_{k}(t)$ with the ordering $x_{k}(t)<x_{k+1}(t)<0$. We can be interested in the label $k$ of the car $x_{k}(t) \leq 0$ which is the closest to the junction point $X=0$. Let us call it $K(t)$. We can normalize the initial data such that $K(0)=0$. Then the quantity of cars that have passed through the junction point $X=0$ during the time interval $[0, t)$ is equal to $-K(t)$, which is the exact discrete analogue of the continuous quantity $-U^{1}(t, 0)$.

On the other hand the number of cars between the positions $a=x_{A}(t)$ and $b=x_{B}(t)$ is obviously equal to $B-A$, and its continuous analogue on the branch $i=m=1$ with $\gamma^{1}=1$, is $\int_{a}^{b} \rho^{1}(t, X) \mathrm{d} X=U^{1}(t, b)-U^{1}(t, a)$. This shows that $U^{1}(t, X)$ can be interpreted as the exact continuous analogue of the discrete labeling of the cars moving in the traffic.

This interpretation is also meaningful on the "exit" branches, i.e. for $j \in\{m+1, \ldots, m+n\}$. Indeed, for such $j$ 's, $U^{j}(t, X)$ is the continuous analogue of the discrete label of the car that have decided to choose the branch $j$ and which is at time $t$ close to the position $X>0$.

\subsection{Getting the Hamilton-Jacobi equations}

We now set

$$
\left\{\begin{array}{lll}
u^{i}(t, X)=-U^{i}(t,-X), & X>0, & \text { for } \quad i=1, \ldots, m \\
u^{j}(t, X)=-U^{j}(t, X), & X>0, & \text { for } \quad j=m+1, \ldots, m+n
\end{array}\right.
$$

and we define the convex Hamiltonians

$$
\begin{cases}H_{i}(p)=-\frac{1}{\gamma^{i}} f^{i}\left(\gamma^{i} p\right) & \text { for } \quad i=1, \ldots, m \\ H_{j}(p)=-\frac{1}{\gamma^{j}} f^{j}\left(-\gamma^{j} p\right) & \text { for } \quad j=m+1, \ldots, m+n .\end{cases}
$$

Then we deduce from (2.5) that we have

$$
u_{t}^{k}+H_{k}\left(u_{X}^{k}\right)=0, \quad X>0, \quad \text { for } \quad k=1, \ldots, m+n
$$

with equality of the functions at the origin, i.e.

$$
u^{k}(t, 0)=u(t, 0) \text { for any } k \in\{1, \ldots, m+n\} .
$$

Notice that for the choice $V_{\max }=1=\rho_{\max }$ in $(2.2)$, we get with $f^{k}(\rho)=f(\rho)=\rho(1-\rho)$ for all $k \in I_{N}$, that

$$
\begin{cases}L_{\mathrm{ref}}(q)=\frac{1}{4}(1+q)^{2} & \\ L_{i}(q)=\frac{1}{\gamma^{2}} L_{\mathrm{ref}}(q) & \text { for } \quad i=1, \ldots, m \\ L_{j}(q)=\frac{1}{\gamma^{j}} L_{\mathrm{ref}}(-q) & \text { for } \quad j=m+1, \ldots, m+n .\end{cases}
$$

In particular this shows that the $L_{k}(0)$ are not all the same, even in the simplest case. 


\subsection{The junction condition and its interpretation}

A junction condition is still needed so that the solution of (2.10) be uniquely defined. Indeed, at first glance, one may think that $u_{t}(t, 0)$ is equal to $-g^{\prime}(t)$ which is given by $(2.6)$ (where we have assumed $(2.7)$ ). The point is that this condition can not be satisfied for every time. One way to be convinced oneself of that fact is to consider the case $m=n=1$ with $f^{1}=f^{2}=f$. Then, we look at solutions $u$ of the Hamilton-Jacobi equation in $\mathbb{R}$ with the artificial junction. We can simply associate with it the classical conservation law on the whole real line. We can then consider a single shock moving with constant velocity for the conservation law. When this shock will pass through the junction point (let us say at time $t_{0}$ ), this will mean that $u_{t}\left(t_{0}, \cdot\right)$ is discontinuous in space at the junction point. In particular the formal computations of Section 2.1 are no longer valid at that time $t_{0}$, even if they are valid for $t \neq t_{0}$. For a general problem, one may expect that our formal computations are only valid for almost every time (even if it is not clear for us).

In view of Theorem 1.4, if we restrict our attention to continuous solutions $u$, then we will have $u \leq u_{\mathrm{oc}}$ where $u_{\mathrm{oc}}$ is the solution associated to the optimal control problem. This shows in particular that we have

$$
u(t, 0) \leq u_{\mathrm{oc}}(t, 0)
$$

which means (in view of (2.8) and the interpretation of $-U^{l}$ given in Sect. 2.1) that we have a universal bound on the total amount of cars passing through the junction point during the time interval $[0, t)$. If we assume moreover that this amount of cars is maximal, then we can choose (and indeed have to choose) $u=u_{\mathrm{oc}}$ and the natural junction condition is then

$$
u_{t}(t, 0)+\max _{k=1, \ldots, N} H_{k}^{-}\left(u_{X}^{k}\left(t, 0^{+}\right)\right)=0
$$

with

$$
H_{k}^{-}(p)=\sup _{q \leq 0}\left(p q-L_{k}(q)\right) \quad \text { and } \quad L_{k}(p)=\sup _{q \in \mathbb{R}}\left(p q-H_{k}(q)\right) .
$$

Using our assumption (2.1) on the functions $f^{k}$, let us define for $k=1, \ldots, N$ the Demand functions

$$
f_{D}^{k}(p)= \begin{cases}f^{k}(p) & \text { for } \quad p \leq \rho_{c}^{k} \\ f^{k}\left(\rho_{c}^{k}\right) & \text { for } \quad p \geq \rho_{c}^{k}\end{cases}
$$

and the Supply functions

$$
f_{S}^{k}(p)= \begin{cases}f^{k}\left(\rho_{c}^{k}\right) & \text { for } \quad p \leq \rho_{c}^{k} \\ f^{k}(p) & \text { for } \quad p \geq \rho_{c}^{k}\end{cases}
$$

From assumption (2.1) on the functions $f^{k}$, we deduce that

$$
\begin{cases}H_{i}^{-}(p)=-\frac{1}{\gamma^{i}} f_{D}^{i}\left(\gamma^{i} p\right), & \text { for } \quad i=1, \ldots, m \\ H_{j}^{-}(p)=-\frac{1}{\gamma^{j}} f_{S}^{j}\left(-\gamma^{j} p\right), & \text { for } \quad j=m+1, \ldots, m+n=N .\end{cases}
$$

Condition (2.11) means that

$$
\begin{aligned}
-U_{t}^{1}(t, 0) & =u_{t}(t, 0)=\min _{k=1, \ldots, N}-H_{k}^{-}\left(u_{X}^{k}\left(t, 0^{+}\right)\right) \\
& =\min \left(\min _{i=1, \ldots, m} \frac{1}{\gamma^{i}} f_{D}^{i}\left(\rho^{i}\left(t, 0^{-}\right)\right), \min _{j=m+1, \ldots, m+n} \frac{1}{\gamma^{j}} f_{S}^{j}\left(\rho^{j}\left(t, 0^{+}\right)\right)\right) .
\end{aligned}
$$

Notice that from (2.7), it is natural to compare

$$
\frac{1}{\gamma^{i}} f^{i}\left(\rho^{i}\left(t, 0^{-}\right)\right) \text {and } \frac{1}{\gamma^{j}} f^{j}\left(\rho^{j}\left(t, 0^{+}\right)\right) .
$$


Then condition (2.12) is nothing else that the Demand and Supply condition of Lebacque, which claims that the passing flux is equal to the minimum between the Demand and the Supply, as it is defined in [23] (at least in the case $m=1$ ).

In the special case $m=1$, it is explained in [24] that this condition (2.12) is also equivalent to the condition defining the Riemann solver at the junction point in the book of Garavello and Piccoli [17]. Let us notice that this condition is also related to the Bardos et al. [5] boundary condition.

\section{Viscosity SOLUtions}

In this section, we consider a weaker assumption than (A1). We introduce the following assumption: (A1') For each $i \in I_{N}$,

- the function $H_{i}: \mathbb{R} \rightarrow \mathbb{R}$ is continuous and $\lim _{|p| \rightarrow+\infty} H_{i}(p)=+\infty$;

- there exists $p_{0}^{i} \in \mathbb{R}$ such that $H_{i}$ is non-increasing on $\left(-\infty, p_{0}^{i}\right]$ and non-decreasing on $\left[p_{0}^{i},+\infty\right)$;

When (A1') holds true, the function $H_{i}^{-}$is defined by $H_{i}^{-}(p)=\inf _{q \leq 0} H_{i}(p+q)$. We now make the following useful remark whose proof is left to the reader.

Lemma 3.1. Assumption (A1) implies Assumption (A1').

Next we give equivalent definitions of viscosity solutions for (1.1). We give a first definition where the junction condition is satisfied in "the classical sense"; we then prove that it is equivalent to impose it in "the generalized sense". It is essential if one expects solutions to be stable.

We give a first definition of viscosity solutions for (1.1) in terms of test functions by imposing the junction condition in the classical sense. We recall the definition of the upper and lower semi-continuous envelopes $u^{*}$ and $u_{*}$ of a function $u:[0, T) \times J$ :

$$
u^{*}(t, x)=\limsup _{(s, y) \rightarrow(t, x)} u(s, y) \quad \text { and } \quad u_{*}(t, x)=\liminf _{(s, y) \rightarrow(t, x)} u(s, y) .
$$

Definition 3.2 (viscosity solutions). A function $u:[0, T) \times J \rightarrow \mathbb{R}$ is a sub-solution (resp. super-solution) of (1.1) on $J_{T}$ if it is upper semi-continuous (resp. lower semi-continuous) and if for any $\phi \in C_{*}^{1}\left(J_{T}\right)$ such that $u \leq \phi$ in $B(P, r)$ for some $P=(t, x) \in J_{T}, r>0$ and such that $u=\phi$ at $P \in J_{T}$, we have

$$
\phi_{t}(t, x)+H\left(x, \phi_{x}(t, x)\right) \leq 0 \quad(\text { resp. } \geq 0),
$$

that is to say

- if $x \in J_{i}^{*}$, then

$$
\phi_{t}(t, x)+H_{i}\left(\phi_{x}(t, x)\right) \leq 0 \quad(\text { resp. } \geq 0)
$$

- if $x=0$, then

$$
\phi_{t}(t, 0)+\max _{i \in I_{N}} H_{i}^{-}\left(\phi_{x}^{i}(t, 0)\right) \leq 0 \quad(\text { resp. } \geq 0) .
$$

A function $u:[0, T) \times J \rightarrow \mathbb{R}$ is a sub-solution (resp. super-solution) of (1.1)-(1.2) on $J_{T}$ if it is a sub-solution (resp. super-solution) of $(1.1)$ on $J_{T}$ and moreover satisfies $u(0, \cdot) \leq u_{0}\left(\right.$ resp. $\left.u(0, \cdot) \geq u_{0}\right)$.

A function $u:[0, T) \times J \rightarrow \mathbb{R}$ is a (viscosity) solution of (1.1) (resp. (1.1)-(1.2)) on $J_{T}$ if $u^{*}$ is a sub-solution and $u_{*}$ is a super-solution of (1.1) (resp. (1.1)-(1.2)) on $J_{T}$.

As mentioned above, the following proposition is important in order to get discontinuous stability results for the viscosity solutions of Definition 3.2.

Proposition 3.3 (equivalence with relaxed junction conditions). Assume (A1'). A function $u: J_{T} \rightarrow \mathbb{R}$ is a sub-solution (resp. super-solution) of (1.1) on $J_{T}$ if and only if for any function $\phi \in C_{*}^{1}\left(J_{T}\right)$ such that $u \leq \phi$ in $J_{T}$ and $u=\phi$ at $(t, x) \in J_{T}$, 
- if $x \in J_{i}^{*}$, then

$$
\phi_{t}(t, x)+H_{i}\left(\phi_{x}(t, x)\right) \leq 0 \quad(\text { resp. } \geq 0) ;
$$

- if $x=0$, then either there exists $i \in I_{N}$ such that

$$
\phi_{t}(t, 0)+H_{i}\left(\phi_{x}(t, 0)\right) \leq 0 \quad(\text { resp. } \geq 0)
$$

or (3.1) holds true.

Proof of Proposition 3.3. We classically reduce to the case where the ball $B(P, r)$ is replaced with $J_{T}$.

The "if" part is clear. Let us prove the "only if" one. We distinguish the sub-solution case and the supersolution one. We start with the super-solutions case since it is slightly easier.

Case 1: super-solution case. We consider a test function $\phi \in C_{*}^{1}\left(J_{T}\right)$ such that $u \geq \phi$ in $J_{T}$ and $u=\phi$ at $\left(t_{0}, x_{0}\right)$. There is nothing to prove if $x_{0} \neq 0$, so we assume $x_{0}=0$. We have to prove that $\phi_{t}\left(t_{0}, 0\right)+$ $\sup _{i \in I_{N}} H_{i}^{-}\left(\phi_{x}^{i}\left(t_{0}, 0\right)\right) \geq 0$. We argue by contradiction and we assume that

$$
\phi_{t}\left(t_{0}, 0\right)+\sup _{i \in I_{N}} H_{i}^{-}\left(\phi_{x}^{i}\left(t_{0}, 0\right)\right)<0 .
$$

Then it is easy to see that there exists a function $\tilde{\phi} \in C_{*}^{1}\left(J_{T}\right)$ such that $\phi \geq \tilde{\phi}$ with equality at the point $\left(t_{0}, 0\right)$ and such that

$$
\tilde{\phi}_{x}^{i}\left(t_{0}, 0\right)=\min \left(\phi_{x}^{i}\left(t_{0}, 0\right), p_{0}^{i}\right) \quad \text { and } \quad \tilde{\phi}_{t}\left(t_{0}, 0\right)=\phi_{t}\left(t_{0}, 0\right) .
$$

Notice that

$$
H_{i}^{-}\left(\tilde{\phi}_{x}^{i}\left(t_{0}, 0\right)\right) \leq H_{i}\left(\tilde{\phi}_{x}^{i}\left(t_{0}, 0\right)\right) \leq H_{i}^{-}\left(\phi_{x}^{i}\left(t_{0}, 0\right)\right) .
$$

The first inequality is straightforward. To check the second inequality, we have to distinguish two cases. Either we have $\tilde{\phi}_{x}^{i}\left(t_{0}, 0\right)<\phi_{x}^{i}\left(t_{0}, 0\right)$, and then $\tilde{\phi}_{x}^{i}\left(t_{0}, 0\right)=p_{0}^{i}$ and we use the fact that the minimum of $H_{i}^{-}$is $H_{i}\left(p_{0}^{i}\right)$. Or $\tilde{\phi}_{x}^{i}\left(t_{0}, 0\right)=\phi_{x}^{i}\left(t_{0}, 0\right)$ and then this common value belongs to the interval $\left(-\infty, p_{0}^{i}\right]$ on which we have $H_{i}=H_{i}^{-}$.

Since $u \geq \tilde{\phi}$ in $J_{T}$ and $u=\tilde{\phi}$ at $\left(t_{0}, 0\right)$, we conclude that either

$$
\tilde{\phi}_{t}\left(t_{0}, 0\right)+\sup _{i \in I_{N}} H_{i}^{-}\left(\tilde{\phi}_{x}^{i}\left(t_{0}, 0\right)\right) \geq 0
$$

or there exists $i \in I_{N}$ such that

$$
\tilde{\phi}_{t}\left(t_{0}, 0\right)+H_{i}\left(\tilde{\phi}_{x}^{i}\left(t_{0}, 0\right)\right) \geq 0 .
$$

In view of (3.3) and (3.4), we obtain a contradiction with (3.2).

Case 2: sub-solution case. We consider a function $\phi \in C^{1}\left(J_{T}\right)$ such that $u \leq \phi$ in $J_{T}$ and $u=\phi$ at $\left(t_{0}, x_{0}\right)$. There is nothing to prove if $x_{0} \neq 0$ and we thus assume $x_{0}=0$. We have to prove that $\phi_{t}\left(t_{0}, 0\right)+$ $\sup _{i \in I_{N}} H_{i}^{-}\left(\phi_{x}^{i}\left(t_{0}, 0\right)\right) \leq 0$. We argue by contradiction and we assume that

$$
\phi_{t}\left(t_{0}, 0\right)+\sup _{i \in I_{N}} H_{i}^{-}\left(\phi_{x}^{i}\left(t_{0}, 0\right)\right)>0 .
$$

In order to construct a test function $\tilde{\phi}$, we first consider $\bar{I}_{1} \subset I_{N}$ the set of $j$ 's such that

$$
H_{j}^{-}\left(\phi_{x}^{j}\left(t_{0}, 0\right)\right)<\sup _{i \in I_{N}} H_{i}^{-}\left(\phi_{x}^{i}\left(t_{0}, 0\right)\right) .
$$

Since $H_{j}$ is coercive, there exists $q^{j} \geq p_{0}^{j}$ such that $H_{j}\left(q^{j}\right)=\sup _{i \in I_{N}} H_{i}^{-}\left(\phi_{x}^{i}\left(t_{0}, 0\right)\right)$.

We next consider a test function $\tilde{\phi} \in C_{*}^{1}\left(J_{T}\right)$ such that $\phi \leq \tilde{\phi}$ with equality at $\left(t_{0}, 0\right)$ and such that

$$
\tilde{\phi}_{x}^{i}\left(t_{0}, 0\right)=\left\{\begin{array}{ll}
\max \left(\phi_{x}^{i}\left(t_{0}, 0\right), q^{i}\right) & \text { if } i \in \bar{I}_{1}, \\
\phi_{x}^{i}\left(t_{0}, 0\right) & \text { if not, }
\end{array} \quad \text { and } \quad \tilde{\phi}_{t}\left(t_{0}, 0\right)=\phi_{t}\left(t_{0}, 0\right) .\right.
$$


Notice that for all $j \in I_{N}$,

$$
H_{j}\left(\tilde{\phi}_{x}^{j}\left(t_{0}, 0\right)\right) \geq \sup _{i \in I_{N}} H_{i}^{-}\left(\tilde{\phi}_{x}^{i}\left(t_{0}, 0\right)\right)=\sup _{i \in I_{N}} H_{i}^{-}\left(\phi_{x}^{i}\left(t_{0}, 0\right)\right)
$$

where for the inequality, we have in particular used the fact that $H_{j}$ is non-decreasing on $\left[p_{0}^{j},+\infty\right)$.

Since $u \leq \tilde{\phi}$ in $J_{T}$ and $u=\tilde{\phi}$ at $\left(t_{0}, 0\right)$, we conclude that either

$$
\tilde{\phi}_{t}\left(t_{0}, 0\right)+\sup _{i \in I_{N}} H_{i}^{-}\left(\tilde{\phi}_{x}^{i}\left(t_{0}, 0\right)\right) \leq 0
$$

or there exists $j \in I_{N}$ such that

$$
\tilde{\phi}_{t}\left(t_{0}, 0\right)+H_{j}\left(\tilde{\phi}_{x}^{j}\left(t_{0}, 0\right)\right) \leq 0 .
$$

In view of (3.6) and (3.7), we obtain a contradiction with (3.5). This ends the proof of the proposition.

We now prove Theorem 1.4.

Proof of Theorem 1.4. Let us consider a function $\phi \in C_{*}^{1}\left(J_{T}\right)$ such that $u \leq \phi$ with equality at $\left(t_{0}, 0\right)$ with $t_{0} \in(0, T)$. Modifying $\phi$ if necessary, we can always assume that the supremum of $u-\phi$ is strict (and reached at $\left.\left(t_{0}, 0\right)\right)$. For $\eta=\left(\eta_{1}, \ldots, \eta_{N}\right) \in\left(\mathbb{R}^{+}\right)^{N}$, we set

$$
M_{\eta}=\sup _{\left(t, x=x_{j} e_{j}\right) \in J_{T}}\left(u(t, x)-\phi(t, x)-\frac{\eta_{j}}{\left|x_{j}\right|}\right) .
$$

Because $u$ is continuous at $\left(t_{0}, 0\right)$, we get for $\eta \in\left(\mathbb{R}_{*}^{+}\right)^{N}$ that

$$
\left\{\begin{array}{l}
M_{\eta} \rightarrow M_{0}=0 \\
\left(t^{\eta}, x^{\eta}\right) \rightarrow\left(t_{0}, 0\right)
\end{array} \mid \quad \text { as soon as one of the component } \eta_{i_{0}} \rightarrow 0 .\right.
$$

where $\left(t^{\eta}, x^{\eta}\right) \in J_{T}$ is a point where the supremum in $M_{\eta}$ is reached.

Moreover given the components $\eta_{j}>0$ for $j \in I_{N} \backslash\left\{i_{0}\right\}$, we can use (3.8) in order to find $\eta_{i_{0}}>0$ small enough to ensure that $x^{\eta} \in J_{i_{0}}^{*}$. Then we have in particular the following sub-solution viscosity inequality at that point $\left(t^{\eta}, x^{\eta}\right)$ :

$$
\phi_{t}+H_{i_{0}}\left(\phi_{x}-\frac{\eta_{i_{0}}}{\left|x^{\eta}\right|^{2}}\right) \leq 0 .
$$

Therefore passing to the limit $\eta_{i_{0}} \rightarrow 0$, we get

$$
\phi_{t}+H_{i_{0}}^{-}\left(\phi_{x}^{i_{0}}\right) \leq 0 \text { at }\left(t_{0}, 0\right) .
$$

Because this is true for any $i_{0} \in I_{N}$, we finally get the sub-solution viscosity inequality at the junction:

$$
\phi_{t}+\max _{i \in I_{N}} H_{i}^{-}\left(\phi_{x}^{i}\right) \leq 0 \quad \text { at } \quad\left(t_{0}, 0\right) .
$$

Now the fact that $u \leq u_{\mathrm{oc}}$ follows from the comparison principle. This ends the proof of the theorem.

\section{The Minimal ACTION}

We already mentioned that the optimal control solution of the Hamilton-Jacobi equation defined by (1.8) plays a central role in our analysis. We remark that for $x \in J$ and $t>0$,

$$
u_{\mathrm{oc}}(t, x)=\inf _{y \in J}\left\{u_{0}(y)+\mathcal{D}(0, y ; t, x)\right\}
$$


where

$$
\mathcal{D}(0, y ; t, x)=\min _{X \in \mathcal{A}(0, y ; t, x)} \int_{0}^{t} L(X(\tau), \dot{X}(\tau)) \mathrm{d} \tau .
$$

More generally, keeping in mind the weak KAM theory, we define the so-called minimal action $\mathcal{D}:\{(s, y, t, x) \in$ $\left.([0, \infty) \times J)^{2}, s<t\right\} \rightarrow \mathbb{R}$ by

$$
\mathcal{D}(s, y ; t, x)=\inf _{X \in \mathcal{A}(s, y ; t, x)} \int_{s}^{t} L(X(\tau), \dot{X}(\tau)) \mathrm{d} \tau
$$

It is convenient to extend $\mathcal{D}$ to $\{s=t\}$. We do so by setting

$$
\mathcal{D}(t, y, t, x)= \begin{cases}0 & \text { if } y=x \\ +\infty & \text { if } y \neq x\end{cases}
$$

Remark 4.1 (dynamic programming principle). Under assumptions (A0)-(A1), it is possible (and easy) to prove the following dynamic programming principle: for all $x \in J$ and $s \in[0, t]$,

$$
u_{\mathrm{oc}}(t, x)=\inf _{y \in J}\left\{u_{\mathrm{oc}}(s, y)+\mathcal{D}(s, y ; t, x)\right\}
$$

Notice that a super-optimality principle will be proved in Proposition 5.1.

The following result can be considered as the core of our analysis. The most important part of the following theorem is the fact that the minimal action is semi-concave with respect to $(t, x)(\operatorname{resp} .(s, y))$.

Theorem 4.2 (key inequalities for $\mathcal{D})$. $\mathcal{D}$ is finite, continuous in $\{(s, y ; t, x): 0<s<t, x, y \in J\}$ and lower semi-continuous in $\{(s, y ; t, x): 0<s \leq t, x, y \in J\}$. Moreover, for all $\left(s_{0}, y_{0}\right)$ and $\left(t_{0}, x_{0}\right) \in(0, T) \times J, s_{0}<t_{0}$, there exist two functions $\phi, \psi \in C_{*}^{1}\left(J_{T}\right)$ and $r>0$ such that

- $\phi \geq \mathcal{D}\left(s_{0}, y_{0} ; \cdot, \cdot\right)$ on a ball $B\left(P_{0}, r\right)$ with equality at $P_{0}=\left(t_{0}, x_{0}\right)$ and

$$
\phi_{t}+H\left(x_{0}, \phi_{x}\right) \geq 0 \quad \text { at } \quad\left(t_{0}, x_{0}\right)
$$

- $\psi \geq \mathcal{D}\left(\cdot, \cdot ; t_{0}, x_{0}\right)$ on a ball $B\left(Q_{0}, r\right)$ with equality at $Q_{0}=\left(s_{0}, y_{0}\right)$ and

$$
\begin{cases}-\psi_{s}+H\left(y_{0},-\psi_{y}\right) \leq 0 \quad \text { at } \quad\left(s_{0}, y_{0}\right) & \text { if } \quad N \geq 2 \\ -\psi_{s}+H_{1}\left(-\psi_{y}\right) \leq 0 \quad \text { at } \quad\left(s_{0}, y_{0}\right) & \text { if } \quad N=1\end{cases}
$$

Moreover, for all $R>0$, there exists a constant $C_{R}>0$ such that we have

$$
d\left(y_{0}, x_{0}\right) \leq R \quad \Longrightarrow \quad\left|\phi_{x}\left(t_{0}, x_{0}\right)\right|+\left|\psi_{y}\left(s_{0}, y_{0}\right)\right| \leq C_{R}
$$

Remark 4.3. As we shall see when proving this result, we can even require equalities instead of inequalities in (4.3) and (4.4).

Since the proof of Theorem 4.2 is lengthy and technical, we postpone it until Section 6 . When proving the main results of our paper in the next section, we also need the following lower bound on $\mathcal{D}$. We remark that this bound ensures in particular that it is finite. 
Lemma 4.4. Assume (A1). Then

$$
\mathcal{D}(s, y ; t, x) \geq \frac{\gamma}{4(t-s)} d(y, x)^{2}-C_{0}(t-s)
$$

where $C_{0}:=\max \left(0,-L_{0}(0)+\frac{\gamma_{0}^{2}}{\gamma}\right)$, $\gamma$ appears in $(A 1), \gamma_{0}=\max _{i \in I_{N}}\left|L_{i}^{\prime}(0)\right|$ and $L_{0}(0)$ is chosen as in $(1.10)$. Moreover,

$$
\mathcal{D}(s, x ; t, x) \leq L_{0}(0)(t-s) .
$$

In particular, if $\left(t_{n}, x_{n}\right) \rightarrow(t, x)$, then $\mathcal{D}\left(t_{n}, x_{n} ; t, x_{n}\right) \rightarrow 0$ as $n \rightarrow \infty$.

Proof of Lemma 4.4. We only prove the first inequality since the remaining of the statement is elementary. We have

$$
L_{i}(p) \geq \frac{\gamma}{2} p^{2}+L_{i}^{\prime}(0) p+L_{i}(0) \geq \frac{\gamma}{2} p^{2}-\gamma_{0}|p|+L_{0}(0) \geq \frac{\gamma}{4} p^{2}+L_{0}(0)-\frac{\gamma_{0}^{2}}{\gamma} .
$$

This shows that

$$
L_{i}(p) \geq \frac{\gamma}{4} p^{2}-C_{0}
$$

Thus we can write for $X(\cdot) \in \mathcal{A}(s, y ; t, x)$,

$$
\int_{s}^{t} L(X(\tau), \dot{X}(\tau)) \mathrm{d} \tau \geq-C_{0}(t-s)+\frac{\gamma}{4} \int_{s}^{t}(\dot{X}(\tau))^{2} \mathrm{~d} \tau
$$

Then Jensen's inequality allows us to conclude.

\section{Proofs of the MAIN RESUlts}

In this section, we investigate the uniqueness of the solution of (1.1)-(1.2). In particular, we will show that the solution constructed by Perron's method coincide with the function $u_{\mathrm{oc}}$ coming from the associated optimal control problem.

\section{1. super-solutions and super-optimality}

In this subsection, we will show that a super-solution satisfies a super-optimality principle. For the sake of clarity, we first give a formal argument to understand this claim. We consider the auxiliary function, for $s \leq t$,

$$
U_{t, x}(s)=\inf _{y \in J}\{u(s, y)+\mathcal{D}(s, y ; t, x)\}
$$

and we are going to explain formally that it is non-decreasing with respect to $s$ as soon as $u$ is a super-solution of (1.1). We call this property a super-optimality principle. Notice that this is strongly related to the fact that the quantity $U_{t, x}(s)$ is constant in $s$ if $u$ is equal to the optimal control solution $u_{\mathrm{oc}}$.

Assume that the infimum defining $U$ is attained for some $\bar{y} \in J$. Then we write

$$
\begin{aligned}
U_{t, x}^{\prime}(s) & =\partial_{s} u(s, \bar{y})+\partial_{s} \mathcal{D}(s, \bar{y} ; t, x) \\
\partial_{x} u(s, \bar{y}) & =-\partial_{y} \mathcal{D}(s, \bar{y} ; t, x) .
\end{aligned}
$$

Moreover assuming $\mathcal{D}$ to be smooth (which is not the case), we formally get from (4.4) the fact that $\partial_{s} \mathcal{D}(s, \bar{y} ; t, x) \geq H\left(\bar{y},-\partial_{y} \mathcal{D}(\bar{s}, \bar{y} ; t, x)\right)$ (at least in the case $\left.N \geq 2\right)$. Hence

$$
U_{t, x}^{\prime}(s) \geq \partial_{s} u(s, \bar{y})+H\left(\bar{y}, \partial_{x} u(s, \bar{y})\right) \geq 0 .
$$

We thus conclude that $U_{t, x}$ is non-decreasing if $u$ is a super-solution of (1.1). We now give a precise statement and a rigorous proof. 
Proposition 5.1 (super-optimality of super-solutions). Assume (A1). Let $u:[0, T) \times J \rightarrow \mathbb{R}$ be a super-solution of (1.1) on $J_{T}$ such that there exists $\sigma>0$ such that for all $(t, x) \in J_{T}$,

$$
u(t, x) \geq-\sigma(1+d(x, 0)) .
$$

Then for all $(t, x) \in J_{T}$ and $s \in(0, t]$,

$$
u(t, x) \geq \inf _{y \in J}\{u(s, y)+\mathcal{D}(s, y ; t, x)\} .
$$

Assume moreover (A0) and that $u$ is a super-solution of (1.1)-(1.2) on $J_{T}$. Then we have $u \geq u_{\mathrm{oc}}$ on $[0, T) \times J$.

Proof of Proposition 5.1. The proof proceeds in several steps.

Step 1: preliminary. Notice first that from (6.7), we get

$$
u(s, y)+\mathcal{D}(s, y ; t, x) \geq \frac{\gamma}{4(t-s)} d(y, x)^{2}-C_{0}(t-s)-\sigma(1+|y|) .
$$

Using the lower semi-continuity of $\mathcal{D}$, we see that the infimum in $y$ of this function is then reached for bounded $y$ 's. Moreover by lower semi-continuity of the map $(s, y ; t, x) \mapsto u(s, y)+\mathcal{D}(s, y ; t, x)$, we deduce in particular that the map $(s ; t, x) \mapsto U_{t, x}(s)$ (and then also $s \mapsto U_{t, x}(s)$ ) is lower semi-continuous.

Step 2: the map $\mathbf{s} \mapsto \mathbf{U}_{\mathbf{t}, \mathbf{x}}(\mathbf{s})$ is non-decreasing. We are going to prove that for $s \in(0, t), U_{t, x}^{\prime}(s) \geq 0$ in the viscosity sense. We consider a test function $\varphi$ touching $U_{t, x}$ from below at $\bar{s} \in(0, t)$. There exists $\bar{y}$ such that

$$
U_{t, x}(\bar{s})=u(\bar{s}, \bar{y})+\mathcal{D}(\bar{s}, \bar{y} ; t, x) .
$$

We deduce from the definition of $U_{t, x}$ that

$$
\varphi(s)-\mathcal{D}(s, y ; t, x)-[\varphi(\bar{s})-\mathcal{D}(\bar{s}, \bar{y} ; t, x)] \leq u(s, y)-u(\bar{s}, \bar{y})
$$

By Theorem 4.2, there exists a test function $\psi$ such that $\psi \geq \mathcal{D}(\cdot, \cdot ; t, x)$ on a ball $B(\bar{Q}, r)$ with equality at $\bar{Q}=(\bar{s}, \bar{y})$. Hence, we can rewrite the previous inequality by replacing $\mathcal{D}$ with $\psi$. We then obtain that $(s, y) \mapsto \varphi(s)-\psi(s, y)$ is a test function touching $u$ at $(\bar{s}, \bar{y})$ from below. Since $u$ is a super-solution of (1.1), we have in the cases $N \geq 2$ or $N=1$ and $\bar{y} \neq 0$

$$
\varphi^{\prime}(\bar{s}) \geq \psi_{s}(\bar{s}, \bar{y})-H\left(\bar{y},-\partial_{y} \psi(\bar{s}, \bar{y})\right) \geq 0
$$

and in the case $N=1$ and $\bar{y}=0$

$$
\varphi^{\prime}(\bar{s}) \geq \psi_{s}(\bar{s}, \bar{y})-H_{1}^{-}\left(-\partial_{y} \psi(\bar{s}, \bar{y})\right) \geq \psi_{s}(\bar{s}, \bar{y})-H_{1}\left(-\partial_{y} \psi(\bar{s}, \bar{y})\right) \geq 0
$$

where we used the properties of the function $\psi$ given by Theorem 4.2 .

Step 3: conclusion. Let us define for $(t, x) \in J_{T}$ the following kind of lower semi-continuous envelope (for the past in time)

$$
\underline{u}(t, x)=\liminf \left\{u\left(t_{n}, x_{n}\right):\left(t_{n}, x_{n}\right) \rightarrow(t, x), t_{n}<t\right\} .
$$

Let us notice that we have

$$
\underline{u}_{*}=u_{*}=u \quad \text { on } \quad J_{T} .
$$

Given a point $(t, x) \in J_{T}$, let us consider a sequence $\left(t_{n}, x_{n}\right) \rightarrow(t, x)$ such that

$$
\underline{u}(t, x)=\lim _{n \rightarrow+\infty} u\left(t_{n}, x_{n}\right) .
$$


Using Lemma 4.4, we have for any $s<t_{n}<t$

$$
U_{t, x_{n}}(s) \leq U_{t, x_{n}}\left(t_{n}\right) \leq u\left(t_{n}, x_{n}\right)+\mathcal{D}\left(t_{n}, x_{n}, t, x_{n}\right) \rightarrow \underline{u}(t, x) .
$$

Therefore from the lower semi-continuity of $U$, we get

$$
U_{t, x}(s) \leq \underline{u}(t, x) .
$$

Again from the lower semi-continuity of the map $(t, x) \mapsto U_{t, x}(s)$, we get passing to the lower semi-continuous envelopes in $(t, x)$ :

$$
U_{t, x}(s) \leq \underline{u}_{*}(t, x)=u(t, x)
$$

where we have used (5.4). This shows (5.3) for $0<s<t$. This is still true for $s=t$ by definition of $\mathcal{D}$. The proof is now complete.

\subsection{Comparison with sub-solutions}

Proposition 5.2 (comparison with sub-solutions). Let $u: J_{T} \rightarrow \mathbb{R}$ be a sub-solution of (1.1)-(1.2) on $J_{T}$, such that there exists $\sigma>0$ such that for all $(t, x) \in J_{T}$,

$$
u(t, x) \leq \sigma(1+d(x, 0)) .
$$

Then we have $u \leq u_{\mathrm{oc}}$ on $J_{T}$.

In order to prove Proposition 5.2, we first state and prove two lemmas.

Lemma 5.3. Assume (A0)-(A1). Then the function $u_{\mathrm{oc}}$ defined in (1.8) satisfies

$$
\left|u_{\mathrm{oc}}(t, x)-u_{0}(x)\right| \leq C t .
$$

Proof of Lemma 5.3. We first get a bound from below. Using (6.7), we deduce (denoting by $L_{u_{0}}$ the Lipschitz constant for $\left.u_{0}\right)$ :

$$
\begin{aligned}
u_{0}(y)+\mathcal{D}(s, y ; t, x) & \geq u_{0}(x)+\frac{\gamma}{4 t}(d(y, x))^{2}-C_{0} t-L_{u_{0}} d(y, x) \\
& \geq u_{0}(x)-C_{2} t
\end{aligned}
$$

with

$$
-C_{2}=\inf _{a \in[0,+\infty)}\left\{\frac{\gamma}{4} a^{2}-C_{0}-L_{u_{0}} a\right\}>-\infty .
$$

This implies that

$$
u_{\mathrm{oc}}(x) \geq u_{0}(x)-C_{2} t
$$

We next get a bound from above. We have

$$
u_{\mathrm{oc}}(x) \leq u_{0}(x)+\mathcal{D}(0, x ; t, x) \leq u_{0}(x)+M t
$$

with

$$
M=\sup _{i \in I_{N}} L_{i}(0)
$$

This ends the proof of the lemma.

Lemma 5.4. Assume (A0)-(A1). Let $u:[0, T) \times J \rightarrow \mathbb{R}$ be a sub-solution of (1.1)-(1.2) on $J_{T}$, satisfying (5.5). Then there exists a constant $C>0$ such that

$$
u(t, x) \leq u_{0}(x)+C t \quad \text { for all } \quad(t, x) \in J_{T} .
$$


Proof of Lemma 5.4. Using the Lipschitz regularity of $u_{0}$, we can easily consider a smooth approximation $u_{0}^{\eta}$ of $u_{0}$ such that $u_{0}^{\eta} \geq u_{0}$ and $\left|u_{0}^{\eta}-u_{0}\right|_{L^{\infty}(J)} \rightarrow 0$ as $\eta \rightarrow 0$. Then consider the following supremum for $\eta, \alpha>0$

$$
N_{\eta, \alpha}=\sup _{(t, x) \in[0, T) \times J}\left\{u(t, x)-u_{0}^{\eta}(x)-C t-\alpha d(x, 0)^{2}-\frac{\eta}{T-t}\right\} .
$$

We claim that $N_{\eta, \alpha} \leq 0$ for some $C$ large enough independent on $\eta, \alpha>0$ small enough. The lemma will be obtained by letting $\alpha$ and $\eta$ go to 0 . We argue by contradiction and assume that $N_{\eta, \alpha}>0$. Thanks to (5.5), the supremum $N_{\eta, \alpha}$ is attained for some $(t, x) \in[0, T) \times J$. If $t=0$, we have $N_{\eta, \alpha} \leq 0$. Therefore $t>0$ and we can use the fact that $u$ is a sub-solution to obtain for $x=x_{i} e_{i}$

$$
\frac{\eta}{T^{2}}+C-\max _{j \in I_{N}} L_{j}(0) \leq \frac{\eta}{T^{2}}+C+H\left(x, \partial_{x} u_{0}^{\eta}(x)+2 \alpha x_{i}\right) \leq 0
$$

where we have used assumption (A1) to estimate $H$ from below. Notice that we have also made use of a slight abuse of notation in the case $x=0$. Choosing $C=\max _{j \in I_{N}}\left|L_{j}(0)\right|$ allows us to conclude to a contradiction. This ends the proof of Lemma 5.4.

We now turn to the proof of Proposition 5.2.

Proof of Proposition 5.2. The proof proceeds in several steps.

Step 1: preliminaries. Let us consider

$$
M=\sup _{(t, x) \in[0, T) \times J}\left\{u(t, x)-u_{\mathrm{oc}}(t, x)\right\} .
$$

From Lemmas 5.3 and 5.4, we deduce that we have $M \leq 2 C T<+\infty$. We want to prove that $M \leq 0$.

To this end, we perform the usual corrections considering the following supremum for $\eta, \alpha>0$

$$
M_{\eta, \alpha}=\sup _{(t, x) \in[0, T) \times J}\left\{u(t, x)-u_{\mathrm{oc}}(t, x)-\alpha d(x, 0)^{2}-\frac{\eta}{T-t}\right\} .
$$

As it is proved classically, we also have that $M_{\eta, \alpha} \rightarrow M_{\eta, 0}$ as $\alpha \rightarrow 0$ where

$$
M_{\eta, 0}=\sup _{(t, x) \in[0, T) \times J}\left\{u(t, x)-u_{\mathrm{oc}}(t, x)-\frac{\eta}{T-t}\right\} .
$$

We argue by contradiction by assuming that $M>0$ and then $M_{\eta, 0} \geq M / 2>0$ for $\eta>0$ small enough and fixed for the rest of the proof.

Step 2: reduction to $\overline{\mathbf{t}}>\mathbf{0}$. Notice that the supremum $M_{\eta, \alpha}$ is achieved for points $(\bar{t}, \bar{x}) \in[0, T) \times J$. Using again Lemmas 5.3 and 5.4 , we also deduce that

$$
M / 2<M_{\eta, 0} \leq M_{\eta, \alpha}+o_{\alpha}(1) \leq 2 C \bar{t}
$$

and hence $\bar{t} \geq \frac{M}{4 C}>0$ for $\alpha$ small enough.

Step 3: a priori bounds. Using the argument of Step 1 of the proof of Proposition 5.1, we see that there exists $\bar{y} \in J$ such that

$$
u_{\mathrm{oc}}(\bar{t}, \bar{x})=u_{0}(\bar{y})+\mathcal{D}(0, \bar{y} ; \bar{t}, \bar{x}) .
$$

Therefore we can rewrite $M_{\eta, \alpha}$ as

$$
M_{\eta, \alpha}=\sup _{0 \leq t<T, x, y \in J}\left\{u(t, x)-u_{0}(y)-\mathcal{D}(0, y ; t, x)-\alpha d(x, 0)^{2}-\frac{\eta}{T-t}\right\},
$$


and the supremum is achieved for $(\bar{t}, \bar{x}, \bar{y}) \in(0, T) \times J^{2}$. Notice that this supremum looks like the classical one for proving the comparison principle for viscosity solutions, with the usual penalization term $(y-x)^{2} / \varepsilon$ replaced here by the function $\mathcal{D}(0, y ; t, x)$.

In view of the bound (6.7) from below on $\mathcal{D}$ and (5.6), we derive from $M_{\eta, \alpha}>0$ that

$$
\frac{\eta}{T-\bar{t}}+\alpha d(\bar{x}, 0)^{2}+\frac{\gamma}{4 \bar{t}} d(\bar{y}, \bar{x})^{2} \leq C_{0} \bar{t}+C \bar{t}+L_{u_{0}} d(\bar{y}, \bar{x})
$$

where $L_{u_{0}}$ denotes the Lipschitz constant of $u_{0}$. We conclude that there exists $C_{T}$ such that

$$
\alpha d(\bar{x}, 0)^{2} \leq C_{T} \quad \text { and } \quad d(\bar{y}, \bar{x}) \leq C_{T}
$$

where $C_{T}$ depends on $T, C_{0}, C, L_{u_{0}}$ and $\gamma$.

Step 4: getting the viscosity inequality. Since $\bar{t}>0$, we have in particular that

$$
u(t, x)-\left(\mathcal{D}(0, \bar{y} ; t, x)+\alpha d(x, 0)^{2}+\frac{\eta}{T-t}\right) \leq u(\bar{t}, \bar{x})-\left(\mathcal{D}(0, \bar{y} ; \bar{t}, \bar{x})+\alpha d(\bar{x}, 0)^{2}+\frac{\eta}{T-\bar{t}}\right) .
$$

By Theorem 4.2 , there exists a test function $\phi$ such that $\phi \geq \mathcal{D}(0, \bar{y} ; \cdot, \cdot)$ on a ball $B(\bar{P}, r)$ with equality at $\bar{P}=(\bar{t}, \bar{x})$. Hence, we can rewrite the previous inequality by replacing $\mathcal{D}$ with $\phi$. We then obtain that $(t, x) \mapsto \phi(t, x)+\alpha d(x, 0)^{2}+\frac{\eta}{T-t}$ touches $u$ from above at $(\bar{t}, \bar{x})$ with $\bar{t}>0$. We use next that $u$ is a sub-solution of (1.1) and get for $\bar{x}=\bar{x}_{i} e_{i}$

$$
\frac{\eta}{T^{2}}+\phi_{t}(\bar{t}, \bar{x})+H\left(\bar{x}, \phi_{x}(\bar{t}, \bar{x})+2 \alpha \bar{x}_{i}\right) \leq 0
$$

where we have made use of a slight abuse of notation in the case $\bar{x}=0$. On the other hand, we have

$$
\phi_{t}(\bar{t}, \bar{x})+H\left(\bar{x}, \phi_{x}(\bar{t}, \bar{x})\right) \geq 0
$$

therefore

$$
\frac{\eta}{T^{2}}+H\left(\bar{x}, \phi_{x}(\bar{t}, \bar{x})+2 \alpha \bar{x}_{i}\right)-H\left(\bar{x}, \phi_{x}(\bar{t}, \bar{x})\right) \leq 0 .
$$

On the one hand, from (5.7), we have $0 \leq \alpha x_{i} \leq \sqrt{\alpha C_{T}}$. On the other hand, we can use (5.7) and (4.5) in order to conclude that

$$
\left|\phi_{x}(\bar{t}, \bar{x})\right| \leq \bar{C}
$$

for some constant $\bar{C}$ which does not depend on $\alpha$. We can now use the fact that the Hamiltonians are locally Lipschitz continuous in order to get the desired contradiction for $\alpha$ small enough. This ends the proof of the proposition.

\subsection{Proof of the main results}

In this subsection, we prove the main results announced in the introduction.

Proof of Theorem 1.2. We simply apply Propositions 5.1 and 5.2 and get $u \leq u_{\mathrm{oc}} \leq v$ which implies the result.

In order to prove Theorem 1.1, we should first prove that solutions are Lipschitz continuous.

Lemma 5.5. Assume (A0)-(A1). Let $u$ be a solution of (1.1)-(1.2) on $J_{T}$. Then $u$ is Lipschitz continuous with respect to $(t, x)$ on $J_{T}$. 
Proof of Lemma 5.5. We first recall (see Lem. 3.1) that (A1) implies (A1'). We know that the solution $u=u^{*}$ given by Theorem A.2 satisfies for some constant $C>0$ and all $(t, x) \in J_{T}$,

$$
\left|u(t, x)-u_{0}(x)\right| \leq C t .
$$

From the comparison principle (Thm. 1.2), we deduce that $u=u^{*} \leq u_{*}$ and then the solution $u=u^{*}=u_{*}$ is continuous.

For $h_{0}>0$ small (with $h_{0}<T$ ), we now consider $h \in\left(0, h_{0}\right)$ and

$$
v(t, x)=u(t+h, x)-\sup _{x \in J}\left(u(h, x)-u_{0}(x)\right) .
$$

This new function satisfies in particular $v(0, x) \leq u_{0}(x)$. Therefore $v$ is a sub-solution of (1.1)-(1.2) on $J_{T-h_{0}}$. We thus conclude from the comparison principle that $v(t, x) \leq u(t, x)$, which implies

$$
u(t+h, x) \leq u(t, x)+C h \text { for all }(t, x) \in J_{T-h_{0}} .
$$

Arguing similarly, we can prove that $u(t+h, x) \geq u(t, x)-C h$. Because $h_{0}$ can be chosen arbitrarily small, we conclude that $u$ is Lipschitz continuous with respect to time on the whole $J_{T}$.

Since $u$ is a viscosity solution of (1.1), it satisfies in particular (in the viscosity sense) for each $i \in I_{N}$ :

$$
H_{i}\left(u_{x}\right) \leq C \text { on }(0, T) \times J_{i}^{*} .
$$

This implies that there exists a constant $\tilde{C}$ such that (in the viscosity sense)

$$
\left|u_{x}\right| \leq \tilde{C} \quad \text { on } \quad(0, T) \times J^{*} .
$$

This implies that $u$ is Lipschitz continuous with respect to the space variable. This ends the proof of the lemma.

We now turn to the proof of Theorem 1.1.

Proof of Theorem 1.1. The uniqueness of the solution follows from Theorem 1.2. The existence is obtained thanks to the optimal control interpretation $\left(u_{\mathrm{oc}}\right.$ is a solution). The Lipschitz regularity was proved in Lemma 5.5 above. The proof of Theorem 1.1 is now complete.

Proof of Theorem 1.3. The fact that the solution is equal to $u_{\mathrm{oc}}$ follows from Propositions 5.1 and 5.2. The representation formula (1.9) follows from (4.1).

\section{A COMPlete STUdy OF THE Minimal ACTION}

\subsection{Reduction of the study}

We start this section with the following remark: the analysis can be reduced to the case $(s, t)=(0,1)$. Precisely, using the fact that the Hamiltonian does not depend on time and is positively homogeneous with respect to the state, the reader can check that a change of variables in time yields the following

Lemma 6.1. For all $x, y \in J$ and $s<t$, we have

$$
\mathcal{D}(s, y ; t, x)=(t-s) \mathcal{D}\left(0, \frac{y}{t-s} ; 1, \frac{x}{t-s}\right) .
$$

This is the reason why we consider the reduced minimal action $\mathcal{D}_{0}: J^{2} \rightarrow \mathbb{R}$ defined by

$$
\mathcal{D}_{0}(y, x)=\mathcal{D}(0, y ; 1, x) .
$$

Thanks to the previous observation, it is enough to prove the following theorem in order to get Theorem 4.2. 
Theorem 6.2 (key equalities for $\left.\mathcal{D}_{0}\right)$. Let us assume $(A 1)$. Then for all $y, x \in J$, the $\mathcal{D}_{0}(y, x)$ is finite. It is continuous in $J^{2}$ and for all $y, x \in J$, there exists a function $\varphi_{0} \in C_{*}^{1}\left(J^{2}\right)$ such that $\varphi_{0} \geq \mathcal{D}_{0}$ on $J^{2}$, $\varphi_{0}(y, x)=\mathcal{D}_{0}(y, x)$ and we have

$$
\begin{aligned}
& \text { if } x \neq 0:\left(\varphi_{0}-x \partial_{x} \varphi_{0}-y \partial_{y} \varphi_{0}\right)(y, x)+H\left(x, \partial_{x} \varphi_{0}(y, x)\right)=0 \\
& \text { if } x=0:\left(\varphi_{0}-x \partial_{x} \varphi_{0}-y \partial_{y} \varphi_{0}\right)(y, 0)+\sup _{i \in I_{N}} H_{i}^{-}\left(\partial_{x}^{i} \varphi_{0}(y, 0)\right)=0
\end{aligned}
$$

and if $y \neq 0$,

$$
\left(\varphi_{0}-x \partial_{x} \varphi_{0}-y \partial_{y} \varphi_{0}\right)(y, x)+H\left(y,-\partial_{y} \varphi_{0}(y, x)\right)=0
$$

and if $y=0$,

$$
\begin{cases}\left(\varphi_{0}-x \partial_{x} \varphi_{0}-y \partial_{y} \varphi_{0}\right)(0, x)+\sup _{j \in I_{N}} H_{j}^{-}\left(-\partial_{y}^{j} \varphi_{0}(0, x)\right)=0 & \text { if } N \geq 2, \\ \left(\varphi_{0}-x \partial_{x} \varphi_{0}-y \partial_{y} \varphi_{0}\right)(0, x)+H_{1}\left(-\partial_{y}^{j} \varphi_{0}(0, x)\right)=0 & \text { if } N=1 .\end{cases}
$$

Moreover, for all $R>0$, there exists $C_{R}>0$ such that for all $x, y \in J$,

$$
d(y, x) \leq R \Rightarrow\left|\partial_{x} \varphi_{0}(y, x)\right|+\left|\partial_{y} \varphi_{0}(y, x)\right| \leq C_{R} .
$$

Remark 6.3. If $I_{0}=I_{N}$, then we have $\mathcal{D}_{0} \in C_{*}^{1}\left(J^{2}\right)$. This good case corresponds to the case where all the $L_{i}(0)$ 's are equal.

We can interpret Lemma 4.4 as follows.

Lemma 6.4. Assume (A1). Then

$$
\mathcal{D}_{0}(y ; x) \geq \frac{\gamma}{4} d(y, x)^{2}-C_{0}
$$

where constants are made precise in Lemma 4.4.

\subsection{Piecewise linear trajectories}

We are going to see that the infimum defining the minimal action can be computed among piecewise linear trajectories, and more precisely among trajectories that are linear as long as they do not reach the junction point. This is a consequence of the fact that the Hamiltonians do not depend on $x$ and are convex (through Jensen's inequality).

In order to state a precise statement, we first introduce that optimal curves are of two types: either they reach the junction point, or they stay in a branch and are straight lines. This is the reason why we introduce first the action associated with straight line trajectories

$$
\mathcal{D}_{\text {straight }}(y, x)= \begin{cases}L_{i}\left(x_{i}-y_{i}\right) & \text { if }(y, x) \in J_{i}^{2} \backslash\{(0,0)\} \\ L_{0}(0) & \text { if } y=0=x \\ +\infty & \text { otherwise }\end{cases}
$$

and the action associated with piecewise linear trajectories passing through the junction point

$$
\mathcal{D}_{\text {junction }}(y, x)=\inf _{0 \leq \tau_{1} \leq \tau_{2} \leq 1}\left\{\mathcal{E}_{1}\left(\tau_{1}, y\right)+\mathcal{E}_{2}\left(\tau_{2}, x\right)\right\}
$$

where

$$
\mathcal{E}_{1}\left(\tau_{1}, y\right)= \begin{cases}\tau_{1} L_{j}\left(-\frac{y_{j}}{\tau_{1}}\right)-\tau_{1} L_{0}(0) & \text { for } y=y_{j} e_{j} \neq 0, \tau_{1} \neq 0 \\ 0 & \text { for } y=0 \\ +\infty & \text { for } \tau_{1}=0, y \neq 0\end{cases}
$$

and

$$
\mathcal{E}_{2}\left(\tau_{2}, x\right)= \begin{cases}\left(1-\tau_{2}\right) L_{i}\left(\frac{x_{i}}{1-\tau_{2}}\right)+\tau_{2} L_{0}(0), & \text { for } x=x_{i} e_{i} \neq 0, \tau_{2} \neq 1 \\ L_{0}(0) & \text { for } x=0 \\ +\infty & \text { for } \tau_{2}=1, x \neq 0\end{cases}
$$


Remark 6.5. By defining the $\mathcal{E}_{i}$ 's in such a way, we treat the degenerate cases: $x=0$ or $y=0$. Indeed, $\tau_{1}$ (resp. $\tau_{2}$ ) measures how long it takes to the trajectory to reach the junction point (resp. the final point $x$ ) from the starting point $y$ (resp. the junction point).

The following facts will be used several times.

Lemma 6.6. The function $\mathcal{E}_{1}$ (resp. $\left.\mathcal{E}_{2}\right)$ is continuous in $(0,1] \times J^{*}\left(\right.$ resp. in $\left.[0,1) \times J^{*}\right)$.

Lemma 6.7. The function $\mathcal{E}_{i}, i=1,2$ are lower semi-continuous in $[0,1] \times J$.

Proof. Consider the function defined for $(\tau, y) \in[0,1] \times J$ by

$$
g(\tau, y)= \begin{cases}\tau L_{i}\left(-\frac{y_{i}}{\tau}\right) & \text { if } y=y_{i} e_{i} \neq 0, \tau \neq 0 \\ \tau L_{0}(0) & \text { if } y=0 \\ +\infty & \text { if } y \neq 0, \tau=0\end{cases}
$$

From the inequality for $\tau>0$ (consequence of (4.6)):

$$
g(\tau, y) \geq \frac{\gamma}{4} \frac{|y|^{2}}{\tau}-C_{0} \tau
$$

we deduce that $g$ is lower semi-continuous. Consequently, the map $\mathcal{E}_{1}$ is lower semi-continuous. We proceed similarly for $\mathcal{E}_{2}$.

We first show the main lemma of this subsection.

Lemma 6.8. The infimum defining the reduced minimal action $\mathcal{D}_{0}$ can be computed among piecewise linear trajectories; more precisely, for all $x, y \in J$,

$$
\mathcal{D}_{0}(y, x)=\min \left(\mathcal{D}_{\text {straight }}(y, x), \mathcal{D}_{\text {junction }}(y, x)\right) .
$$

Proof. We write with obvious notation $\mathcal{D}_{0}(y, x)=\inf _{X \in \mathcal{A}_{0}(y, x)} \mathcal{E}(X)$. In order to prove the lemma, it is enough to consider a curve $X \in \mathcal{A}_{0}(y, x)$ and prove that

$$
\mathcal{E}(X) \geq \min \left(\mathcal{D}_{\text {straight }}(y, x), \mathcal{D}_{\text {junction }}(y, x)\right) .
$$

To do so, we first remark that the uniform convexity of $L_{i}$ implies that for all $p_{0} \in \mathbb{R}$, we have

$$
L_{i}(p) \geq L_{i}\left(p_{0}\right)+L_{i}^{\prime}\left(p_{0}\right)\left(p-p_{0}\right)+\frac{\gamma}{2}\left(p-p_{0}\right)^{2} .
$$

We now consider an admissible trajectory $X:[0,1] \rightarrow J$ and we treat different cases.

Case A: $X\left(\left(t_{1}, t_{2}\right)\right) \subset J_{i}^{*}$. We assume that a curve $X$ stays in one of the branch $J_{i}^{*}$ on the time interval $\left(t_{1}, t_{2}\right)$ with $t_{1}<t_{2}$. In such a case, we consider the curve $\tilde{X}$ with same end points $X\left(t_{1}\right)$ and $X\left(t_{2}\right)$ in $J_{i}$ but linear. If $p_{0} \in \mathbb{R}$ is such that $p_{0} e_{i}=\dot{\tilde{X}}(\tau)$ for $\tau \in\left(t_{1}, t_{2}\right)$ and $p e_{i}=\dot{X}(\tau)$, we deduce from (6.8) that

$$
\int_{t_{1}}^{t_{2}} L(X(\tau), \dot{X}(\tau)) \mathrm{d} \tau \geq \int_{t_{1}}^{t_{2}} L(\tilde{X}(\tau), \dot{\tilde{X}}(\tau)) \mathrm{d} \tau+\frac{\gamma}{2} \int_{t_{1}}^{t_{2}}|\dot{X}(\tau)-\dot{\tilde{X}}(\tau)|^{2} \mathrm{~d} \tau .
$$

Case B: $X\left(\left[t_{1}, t_{2}\right]\right) \subset J_{i}$ with $X\left(t_{1}\right)=X\left(t_{2}\right)=0$. In that case, let us set $\tilde{X}(\tau)=0$ for $\tau \in\left[t_{1}, t_{2}\right]$. Using (6.8) with $p_{0}=0$ and the definition of $L_{0}$ as a minimum of the $L_{j}$ 's (see (1.10)), we get that

$$
L_{i}(p) \geq L_{0}(0)+L_{i}^{\prime}(0) p+\frac{\gamma}{2} p^{2}
$$

from what we deduce that (6.9) still holds true. 
Case C: the general case. By assumption, we have $X \in \mathcal{A}_{0}(y ; x) \subset C([0,1])$. We then distinguish two cases. Either $0 \notin X([0,1])$, and then we define $\tilde{X}$ as in Case A. In this case, (6.9) implies that

$$
\mathcal{E}(X) \geq \mathcal{D}_{\text {straight }}(y, x) .
$$

Or $0 \in X([0,1])$, and then we call $\left[\tau_{1}, \tau_{2}\right] \subset[s, t]$ the largest interval such that $X\left(\tau_{1}\right)=0=X\left(\tau_{2}\right)$, and define $\tilde{X}$ as follows: it is linear between 0 and $\tau_{1}$, and reaches 0 at $\tau_{1}$; it stays at 0 in $\left(\tau_{1}, \tau_{2}\right)$; then it is linear in $\left(\tau_{2}, 1\right)$ and reaches $x$ at $t=1$. Using again the continuity of $X$, we can find a decomposition of $\left[\tau_{1}, \tau_{2}\right]$ as a disjoint union of intervals $\mathcal{I}_{k}$ (with an at most countable union)

$$
\left[\tau_{1}, \tau_{2}\right]=\bigcup_{k} \mathcal{I}_{k}
$$

such that for each $k, X\left(\mathcal{I}_{k}\right) \subset J_{i_{k}}$ for some $i_{k} \in I_{N}$ and $X=0$ on $\partial \mathcal{I}_{k}$. Using Case A or Case B on each segment $\overline{\mathcal{I}}_{k}$, we deduce that

$$
\mathcal{E}(X) \geq \mathcal{D}_{\text {junction }}(y, x)
$$

\subsection{Continuity of the (reduced) minimal action}

Lemma 6.9 (continuity of $\mathcal{D}_{\text {junction }}$ ). The function $\mathcal{D}_{\text {junction }}$ is continuous in $J^{2}$.

Proof. We first prove that $\mathcal{D}_{\text {junction }}$ is lower semi-continuous. We know from Lemma 6.7 that the function

$$
G\left(\tau_{1}, \tau_{2} ; y ; x\right)=\mathcal{E}_{1}\left(\tau_{1}, y\right)+\mathcal{E}_{2}\left(\tau_{2}, x\right)
$$

is lower semi-continuous for $y, x \in J$ and $0 \leq \tau_{1} \leq \tau_{2} \leq 1$. Therefore the function

$$
\mathcal{D}_{\text {junction }}(y ; x)=\inf _{0 \leq \tau_{1} \leq \tau_{2} \leq 1} G\left(\tau_{1}, \tau_{2} ; y, x\right)
$$

is also lower semi-continuous (since the infimum is taken over a compact set). Besides, the infimum is in fact a minimum.

We now prove that $\mathcal{D}_{\text {junction }}$ is upper semi-continuous at any point $(y, x)$. Consider first $\left(\tau_{1}, \tau_{2}\right) \in[0,1]^{2}$ such that

$$
\mathcal{D}_{\text {junction }}(y, x)=\mathcal{E}_{1}\left(\tau_{1}, y\right)+\mathcal{E}_{2}\left(\tau_{2}, x\right) .
$$

Given any sequence $\left(y^{k}, x^{k}\right) \rightarrow(y, x)$, we want to show that

$$
\mathcal{D}_{\text {junction }}\left(y^{k}, x^{k}\right) \leq \mathcal{D}_{\text {junction }}(y, x)+o_{k}(1) .
$$

We use

$$
\mathcal{D}_{\text {junction }}\left(y^{k}, x^{k}\right) \leq \mathcal{E}_{1}\left(\tau_{1}^{k}, y^{k}\right)+\mathcal{E}_{2}\left(\tau_{2}^{k}, x^{k}\right)
$$

with an appropriate choice of $\left(\tau_{1}^{k}, \tau_{2}^{k}\right)$.

Case 1: $y \in J_{j}^{*}, x \in J_{i}^{*}$. In this case, we choose $\left(\tau_{1}^{k}, \tau_{2}^{k}\right)=\left(\tau_{1}, \tau_{2}\right) \in(0,1)^{2}$ and we use Lemma 6.6 in order to get

$$
\mathcal{E}_{1}\left(\tau_{1}^{k}, y^{k}\right) \rightarrow \mathcal{E}_{1}\left(\tau_{1}, y\right)
$$

and

$$
\mathcal{E}_{2}\left(\tau_{2}^{k}, x^{k}\right) \rightarrow \mathcal{E}_{2}\left(\tau_{2}, x\right)
$$

Hence we conclude that (6.10) holds true. 
Case 2: $y=0, x \in J_{i}^{*}$. We choose $\left(\tau_{1}^{k}, \tau_{2}^{k}\right)=\left(\left|y^{k}\right|, \max \left(\tau_{2},\left|y^{k}\right|\right)\right) \in[0,1)^{2}$. We still have $\tau_{2}^{k} \rightarrow \tau_{2}$ and we can use Lemma 6.6 in order to get

$$
\mathcal{E}_{2}\left(\tau_{2}^{k}, x^{k}\right) \rightarrow \mathcal{E}_{2}\left(\tau_{2}, x\right)
$$

We also have (if $y^{k} \in J_{j}$ )

$$
\mathcal{E}_{1}\left(\tau_{1}^{k}, y^{k}\right) \leq\left|y^{k}\right| L_{j}\left(-\frac{y_{j}^{k}}{\left|y^{k}\right|}\right)-\left|y^{k}\right| L_{0}(0) \rightarrow 0=\mathcal{E}_{1}\left(\tau_{1}, 0\right) .
$$

Hence we conclude that (6.10) holds true.

Case 3: $y \in J_{j}^{*}, x=0$. We choose $\left(\tau_{1}^{k}, \tau_{2}^{k}\right)=\left(\min \left(\tau_{1}, 1-\left|x^{k}\right|\right), 1-\left|x^{k}\right|\right) \in(0,1]^{2}$ We still have $\tau_{1}^{k} \rightarrow \tau_{1}$ and then

$$
\mathcal{E}_{1}\left(\tau_{1}^{k}, y^{k}\right) \rightarrow \mathcal{E}_{1}\left(\tau_{1}, y\right)
$$

( since $\mathcal{E}_{1}$ is continuous in $\left.(0,1] \times J^{*}\right)$. We also have (if $x^{k} \in J_{i}$ )

$$
\mathcal{E}_{2}\left(\tau_{2}^{k}, x^{k}\right) \leq\left|x^{k}\right| L_{i}\left(\frac{x_{i}^{k}}{\left|x^{k}\right|}\right)+\left(1-\left|x^{k}\right|\right) L_{0}(0) \rightarrow L_{0}(0)=\mathcal{E}_{2}\left(\tau_{2}, 0\right) .
$$

Hence we conclude that (6.10) holds true.

Case 4: $y=0, x=0$. We choose $\left(\tau_{1}^{k}, \tau_{2}^{k}\right)=\left(\left|y^{k}\right|, 1-\left|x^{k}\right|\right) \in[0,1) \times(0,1]$. We deduce (6.10) from (6.11) and (6.12).

Lemma 6.10. The function $\mathcal{D}_{0}$ is continuous in $J^{2}$.

Proof. Since $\mathcal{D}_{\text {straight }}$ is lower semi-continuous, we can use Lemmas 6.8 and 6.9 in order to conclude that $\mathcal{D}_{0}$ is lower semi-continuous.

Consider $(y, x) \in \partial\left(J_{i} \times J_{i}\right) \backslash\{(0,0)\}$. Then either $x=0$ or $y=0$. Moreover for $y=y_{i} e_{i}$ and $x=x_{i} e_{i}$,

$$
\mathcal{D}_{\text {junction }}(y, x) \leq\left\{\begin{array}{l}
\mathcal{E}_{1}(1, y)+\mathcal{E}_{2}(1, x) \text { if } x_{i}=0 \\
\mathcal{E}_{1}(0, y)+\mathcal{E}_{2}(0, x) \text { if } y_{i}=0
\end{array}\right\} \leq L_{i}\left(x_{i}-y_{i}\right) .
$$

Therefore for each $i \in I_{N}$, we have for $(y, x) \in \partial\left(J_{i} \times J_{i}\right)$,

$$
\mathcal{D}_{\text {junction }}(y, x) \leq \mathcal{D}_{\text {straight }}(y, x) .
$$

Therefore we have with $y=y_{i} e_{i}, x=x_{i} e_{i}$

$$
\mathcal{D}_{0}(y, x)= \begin{cases}\min \left(\mathcal{D}_{\text {junction }}(y, x), L_{i}\left(x_{i}-y_{i}\right)\right) & \text { if }(y, x) \in J_{i} \times J_{i} \\ \mathcal{D}_{\text {junction }}(y, x) & \text { if }(y, x) \in \partial\left(J_{i} \times J_{i}\right) \\ \mathcal{D}_{\text {junction }}(y, x) & \text { otherwise. }\end{cases}
$$

This implies that $\mathcal{D}_{0}$ is continuous in $J^{2}$.

\subsection{Study of $\mathcal{D}_{\text {junction }}$}

In view of (6.13), we see that the study of $\mathcal{D}_{0}$ can now be reduced to the study of $\mathcal{D}_{\text {junction }}$. The function $\mathcal{D}_{\text {junction }}$ is defined as a minimum over a triangle $\left\{\left(\tau_{1}, \tau_{2}\right) \in[0,1]^{2}: \tau_{1} \leq \tau_{2}\right\}$. We will see below that $\mathcal{D}_{\text {junction }}$ is defined implicitly when the constraint $\tau_{1} \leq \tau_{2}$ is active $\left(\mathcal{D}_{\text {implicit }}\right)$ or defined explicitly if not $\left(\mathcal{D}_{\text {linear }}\right)$. In other words, it will be linear "as long as" trajectories stay some time $\left(\tau_{2}-\tau_{1}>0\right)$ at the junction point.

We first define for $(y, x) \in J^{2}$,

$$
\mathcal{D}_{\text {implicit }}(y, x)=\inf _{0 \leq \tau \leq 1}\left\{\mathcal{E}_{1}(\tau, y)+\mathcal{E}_{2}(\tau, x)\right\} .
$$

The continuity of $\mathcal{D}_{\text {implicit }}$ will be used later on. 
Lemma 6.11 (continuity of $\mathcal{D}_{\text {implicit }}^{j i}$ ). The restrictions $\mathcal{D}_{\text {implicit }}^{j i}$ of $\mathcal{D}_{\text {implicit }}$ are continuous in $\left(J_{j} \times J_{i}\right) \backslash\{(0,0)\}$ and continuous at $(0,0)$ if $j \in I_{0}$ or $i \in I_{0}$.

Proof of Lemma 6.11. From Lemma 6.7, we deduce that $\mathcal{D}_{\text {implicit }}^{j i}$ is lower semi-continuous on $J_{j} \times J_{i}$. We now show that $\mathcal{D}_{\text {implicit }}^{j i}$ is upper semi-continuous at any point $(y, x) \in\left(J_{j} \times J_{i}\right) \backslash\{(0,0)\}$ and also at $(0,0)$ if $j \in I_{0}$ or $i \in I_{0}$. We first consider $\tau \in[0,1]$ such that

$$
\mathcal{D}_{\text {implicit }}^{j i}(y, x)=\mathcal{E}_{1}(\tau, y)+\mathcal{E}_{2}(\tau, x) \quad \text { with } \quad 0 \leq \tau \leq 1 .
$$

For any sequence $\left(y^{k}, x^{k}\right) \rightarrow(y, x)$ with $\left(y^{k}, x^{k}\right) \in J_{j} \times J_{i}$, we want to show that

$$
\mathcal{D}_{\text {implicit }}^{j i}\left(y^{k}, x^{k}\right) \leq \mathcal{D}_{\text {implicit }}^{j i}(y, x)+o_{k}(1) .
$$

Arguing as in Lemma 6.9, we use

$$
\mathcal{D}_{\text {implicit }}^{j i}\left(y^{k}, x^{k}\right) \leq \mathcal{E}_{1}\left(\tau^{k}, y^{k}\right)+\mathcal{E}_{2}\left(\tau^{k}, x^{k}\right)
$$

and we choose $\tau^{k}$ as follows

$$
\begin{array}{ll}
\text { if } y \in J_{j}^{*}, x \in J_{i}^{*}: & \tau^{k}=\tau \in(0,1), \\
\text { if } y=0, x \in J_{i}^{*}: & \tau^{k}=\left|y_{k}\right| \in[0,1), \\
\text { if } y \in J_{j}^{*}, x=0: & \tau^{k}=1-\left|x^{k}\right| \in(0,1], \\
\text { if } x=0, j \in I_{0}: & \tau^{k}=1-\left|x^{k}\right| \in(0,1], \\
\text { if } y=0, x=0, i \in I_{0}: & \tau^{k}=\left|y^{k}\right| \in[0,1) .
\end{array}
$$

This ends the proof of the lemma.

We next define for $(y, x) \in J^{j} \times J^{i}$

$$
\mathcal{D}_{\text {linear }}^{j i}(y, x)=-L_{j}^{\prime}\left(\xi_{j}^{-}\right) y+L_{i}^{\prime}\left(\xi_{i}^{+}\right) x+L_{0}(0)
$$

where $\xi_{l}^{ \pm}$are defined thanks to the following function (for $l \in I_{N}$ )

$$
K_{l}(\xi)=L_{l}(\xi)-\xi L_{l}^{\prime}(\xi)-L_{0}(0)
$$

Precisely, $\xi_{l}^{ \pm}=\left(K_{l}^{ \pm}\right)^{-1}(0) \neq 0$ when $l \notin I_{0}$ (see Lem. 6.17 below). We will see that $K_{l}$ plays an important role in the analysis of $\mathcal{D}_{\text {junction. }}$. In particular, it allows us to define, when $i \notin I_{0}$ and $j \notin I_{0}$, the following convex subset (triangle) of $J_{j} \times J_{i}$ :

$$
\Delta^{j i}=\left\{(y, x) \in J_{j} \times J_{i}, \quad \frac{x}{\xi_{i}^{+}}-\frac{y}{\xi_{j}^{-}}<1\right\} .
$$

It is convenient to set $\Delta^{j i}=\emptyset$ if $i \in I_{0}$ or $j \in I_{0}$. We next state a series of lemmas before proving them.

Lemma 6.12 (link between $\mathcal{D}_{\text {junction }}, \mathcal{D}_{\text {linear }}, \mathcal{D}_{\text {implicit }}$ ).

$$
\mathcal{D}_{\text {junction }}^{j i}(y, x)=\left\{\begin{array}{lll}
\mathcal{D}_{\text {linear }}^{j i}(y, x) & \text { if } \quad(y, x) \in \Delta^{j i} \\
\mathcal{D}_{\text {implicit }}^{j i}(y, x) & \text { if } \quad(y, x) \in\left(J_{j} \times J_{i}\right) \backslash \Delta^{j i} .
\end{array}\right.
$$

Lemma 6.13 (the equations in the interior). The functions $\mathcal{D}_{\text {junction }}^{j i}, \mathcal{D}_{\text {linear }}^{j i}$ and $\mathcal{D}_{\text {implicit }}^{j i}$ are convex and $C^{1}$ in $J_{j}^{*} \times J_{i}^{*}$ and, if $\tilde{\mathcal{D}}$ is one of them, it satisfies for $(y, x) \in J_{j}^{*} \times J_{i}^{*}$

$$
\left\{\begin{array}{l}
\tilde{\mathcal{D}}(y, x)-x \partial_{x} \tilde{\mathcal{D}}(y, x)-y \partial_{y} \tilde{\mathcal{D}}(y, x)+H_{i}\left(\partial_{x} \tilde{\mathcal{D}}(y, x)\right)=0, \\
\tilde{\mathcal{D}}(y, x)-x \partial_{x} \tilde{\mathcal{D}}(y, x)-y \partial_{y} \tilde{\mathcal{D}}(y, x)+H_{j}\left(-\partial_{y} \tilde{\mathcal{D}}(y, x)\right)=0 .
\end{array}\right.
$$


Lemma 6.14 (study of $\mathcal{D}_{\text {implicit }}$ ). For $(y, x) \in J_{j}^{*} \times J_{i}^{*}$, there exists a unique $\tau=T(y, x) \in(0,1)$ such that

$$
\mathcal{D}_{\text {implicit }}^{j i}(y, x)=\mathcal{E}_{1}(\tau, y)+\mathcal{E}_{2}(\tau, x) .
$$

Moreover,

$$
\left\{\begin{array}{lll}
\partial_{x} \mathcal{D}_{\text {implicit }}^{j i}(y, x)=L_{i}^{\prime}\left(\xi_{x}\right) & \text { with } & \xi_{x}=\frac{x}{1-T(y, x)}, \\
\partial_{y} \mathcal{D}_{\text {implicit }}^{j i}(y, x)=-L_{j}^{\prime}\left(\xi_{y}\right) & \text { with } & \xi_{y}=-\frac{y}{T(y, x)}
\end{array}\right.
$$

Lemma 6.15 (study of $T$ ). For $(y, x) \in\left(J_{j} \times J_{i}\right) \backslash\{(0,0)\}$, there is a unique $\tau=T(y, x) \in[0,1]$ such that

$$
\mathcal{D}_{\text {implicit }}^{j i}(y, x)=\mathcal{E}_{1}(\tau, y)+\mathcal{E}_{2}(\tau, x) .
$$

Moreover $T \in C\left(J_{j} \times J_{i} \backslash\{(0,0)\}\right)$ and

$$
T(y, x)= \begin{cases}\max \left(0,1-\frac{x}{\xi_{i}^{+}}\right) & \text {if } \quad(y, x) \in\left(\{0\} \times J_{i}^{*}\right) \backslash \Delta^{j i}, \\ \min \left(1,-\frac{y}{\xi_{j}^{-}}\right) & \text {if } \quad(y, x) \in\left(J_{j}^{*} \times\{0\}\right) \backslash \Delta^{j i} .\end{cases}
$$

Lemma 6.16 ( $\mathcal{D}_{\text {junction }}^{j i}$ at the boundary). Then we have $\mathcal{D}_{\text {junction }}^{j i} \in C^{1}\left(J_{j} \times J_{i}\right)$ with

$$
\left\{\begin{array}{l}
\partial_{x} \mathcal{D}_{\text {junction }}^{j i}(y, x)=L_{i}^{\prime}\left(\xi_{x}\right) \\
\partial_{y} \mathcal{D}_{\text {junction }}^{j i}(y, x)=-L_{j}^{\prime}\left(\xi_{y}\right)
\end{array}\right.
$$

where $\xi_{y} \leq 0 \leq \xi_{x}$ satisfy

$$
\left(\xi_{x}, \xi_{y}\right)= \begin{cases}\left(\max \left(x, \xi_{i}^{+}\right),\left(K_{j}^{-}\right)^{-1}\left(K_{i}\left(\xi_{x}\right)\right)\right) & \text { if }(y, x) \in\left(\{0\} \times J_{i}\right) \backslash \Delta^{j i} \\ \left(\xi_{i}^{+}, \xi_{j}^{-}\right) & \text {if }(y, x) \in\left(\{0\} \times J_{i}\right) \cap \Delta^{j i} \\ \left(\left(K_{i}^{+}\right)^{-1}\left(K_{j}\left(\xi_{y}\right)\right),-\max \left(y,-\xi_{j}^{-}\right)\right) & \text {if }(y, x) \in\left(J_{j} \times\{0\}\right) \backslash \Delta^{j i} \\ \left(\xi_{i}^{+}, \xi_{j}^{-}\right) & \text {if }(y, x) \in\left(J_{j} \times\{0\}\right) \cap \Delta^{j i}\end{cases}
$$

Moreover we have

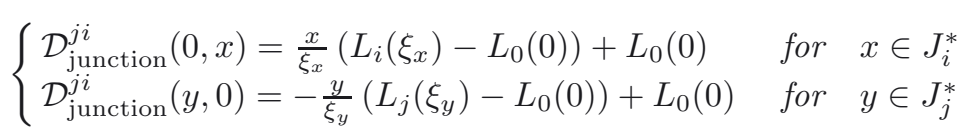

and

$$
\begin{aligned}
\mathcal{D}_{\text {junction }}^{j i}(x, y)-x \partial_{x} \mathcal{D}_{\text {junction }}^{j i}(x, y)-y \partial_{y} \mathcal{D}_{\text {junction }}^{j i}(x, y) & \\
& = \begin{cases}L_{0}(0)+K_{i}\left(\max \left(x, \xi_{i}^{+}\right)\right) & \text {if } \quad(y, x) \in\{0\} \times J_{i}, \\
L_{0}(0)+K_{j}\left(-\max \left(y,-\xi_{j}^{-}\right)\right) & \text {if } \quad(y, x) \in J_{j} \times\{0\} .\end{cases}
\end{aligned}
$$

Before proving these lemmas, the reader can check the following useful properties of the function $K_{l}$ that will be used in their proofs.

Lemma 6.17 (properties of $K_{l}$ ). Assume (A1). Then for any $l \in I_{N}$, we have

$$
\begin{aligned}
K_{l}^{\prime}(\xi) \geq \gamma|\xi| & \text { for } \quad \xi \in(-\infty, 0), \\
K_{l}^{\prime}(\xi) \leq-\gamma|\xi| & \text { for } \quad \xi \in(0,+\infty) .
\end{aligned}
$$

We define $\left(K_{l}^{-}\right)^{-1}$ as the inverse of the function $K_{l}$ restricted to $(-\infty, 0]$, and $\left(K_{l}^{+}\right)^{-1}$ as the inverse of the function $K_{l}$ restricted to $[0,+\infty)$. We set

$$
\xi_{l}^{ \pm}=\left(K_{l}^{ \pm}\right)^{-1}(0)
$$


Then we have

$$
\begin{array}{ll} 
\pm \xi_{l}^{ \pm}=0 & \text { if } \quad l \in I_{0}, \\
\pm \xi_{l}^{ \pm}>0 & \text { if } \quad l \in I_{N} \backslash I_{0} .
\end{array}
$$

Moreover we have

$$
K_{l}(\xi)=-H_{l}\left(L_{l}^{\prime}(\xi)\right)-L_{0}(0)
$$

Proof of Lemmas 6.12-6.14. The proof proceeds in several steps.

Step 1: first study of $\mathcal{D}_{\text {junction. }}^{j i}$ Let us define

$$
G\left(\tau_{1}, \tau_{2}, y, x\right)=\mathcal{E}_{1}\left(\tau_{1}, y\right)+\mathcal{E}_{2}\left(\tau_{2}, x\right)
$$

For $\tau_{1}, \tau_{2} \in(0,1)$, and setting

$$
\xi_{y}=-\frac{y}{\tau_{1}}, \quad \xi_{x}=\frac{x}{1-\tau_{2}}
$$

and $V_{y}=\left(\xi_{y}, 0,1,0\right)$ and $V_{x}=\left(0, \xi_{x}, 0,1\right)$, we compute

$$
D^{2} G\left(\tau_{1}, \tau_{2}, y, x\right)=\frac{L_{j}^{\prime \prime}\left(\xi_{y}\right)}{\tau_{1}} V_{y}^{T} V_{y}+\frac{L_{i}^{\prime \prime}\left(\xi_{x}\right)}{1-\tau_{2}} V_{x}^{T} V_{x} \geq 0
$$

Therefore $G$ is in particular convex on $(0,1) \times(0,1) \times J_{j}^{*} \times J_{i}^{*}$. Because $G$ is in particular lower semi-continuous on $[0,1] \times[0,1] \times J_{j}^{*} \times J_{i}^{*}$, and

$$
G\left(0, \tau_{2}, y, x\right)=+\infty=G\left(\tau_{1}, 1, y, x\right) \quad \text { for }(y, x) \in J_{j}^{*} \times J_{i}^{*},
$$

we deduce that

$$
\mathcal{D}_{\text {junction }}^{j i}(y, x)=\inf _{0<\tau_{1} \leq \tau_{2}<1} G\left(\tau_{1}, \tau_{2}, y, x\right) \quad \text { for } \quad(y, x) \in J_{j}^{*} \times J_{i}^{*} .
$$

This implies that $\mathcal{D}_{\text {junction }}^{j i}$ is also convex in $J_{j}^{*} \times J_{i}^{*}$. Notice that in particular

$$
D_{\tau_{1} \tau_{1}}^{2} G\left(\tau_{1}, \tau_{2}, y, x\right)=\frac{y^{2}}{\tau_{1}^{3}} L_{j}^{\prime \prime}\left(\xi_{y}\right)>0
$$

and

$$
D_{\tau_{2} \tau_{2}}^{2} G\left(\tau_{1}, \tau_{2}, y, x\right)=\frac{x^{2}}{\left(1-\tau_{2}\right)^{3}} L_{i}^{\prime \prime}\left(\xi_{x}\right)>0 .
$$

The map $\left(\tau_{1}, \tau_{2}\right) \mapsto G\left(\tau_{1}, \tau_{2}, y, x\right)$ is then strictly convex on the convex set

$$
\left\{\left(\tau_{1}, \tau_{2}\right) \in(0,1)^{2}, \quad \tau_{1} \leq \tau_{2}\right\} .
$$

Therefore using again (6.25) and the lower semi-continuity of $G$, we deduce that it has a unique minimum that we denote by $\left(\tau_{1}, \tau_{2}\right)$ satisfying $0<\tau_{1} \leq \tau_{2}<1$.

Step 2: study of $\mathcal{D}_{\text {implicit }}^{j i}$. Let us consider the following function

$$
e(\tau, y, x)=G(\tau, \tau, y, x)
$$

For $\tau \in(0,1)$, setting

$$
\xi_{y}=-\frac{y}{\tau}, \quad \xi_{x}=\frac{x}{1-\tau}
$$


and proceeding similarly as in Step 1, we can deduce that

$$
\mathcal{D}_{\text {implicit }}^{j i}(y, x)=\inf _{\tau \in(0,1)} e(\tau, y, x) \quad \text { for } \quad(y, x) \in J_{j}^{*} \times J_{i}^{*}
$$

and that $\mathcal{D}_{\text {implicit }}^{j i}$ is also convex on $J_{j}^{*} \times J_{i}^{*}$. We can also deduce that the map $\tau \mapsto e(\tau, y, x)$ is strictly convex on $(0,1)$ for $(y, x) \in J_{j}^{*} \times J_{i}^{*}$ and that it has a unique minimum that we denote by $\tau \in(0,1)$ such that

$$
\mathcal{D}_{\text {implicit }}^{j i}(y, x)=e(\tau, y, x) .
$$

Using the derivative with respect to $\tau$, we see that $\tau$ is characterized by the equation

$$
F=0 \quad \text { with } \quad F(\tau, y, x):=K_{j}\left(-\frac{y}{\tau}\right)-K_{i}\left(\frac{x}{1-\tau}\right) .
$$

Moreover

$$
\partial_{\tau} F(\tau, y, x)=D_{\tau \tau}^{2} e(\tau, y, x)>0 .
$$

Using the regularity $C^{2}$ of $L_{l}$ given in assumption (A1), we see that the unique solution $\tau=T(y, x)$ of $F(\tau, y, x)=$ 0 is continuously differentiable with respect to $(y, x)$. Therefore we deduce that $\mathcal{D}_{\text {implicit }}^{j i} \in C^{1}\left(J_{j}^{*} \times J_{i}^{*}\right)$.

We have

$$
\begin{aligned}
\mathcal{D}_{\text {implicit }}^{j i}(y, x) & =\mathcal{E}_{1}(T(y, x), y)+\mathcal{E}_{2}(T(y, x), x), \\
\partial_{y} \mathcal{D}_{\text {implicit }}^{j i}(y, x) & =\left(\partial_{y} \mathcal{E}_{1}\right)(T(y, x), y)=-L_{j}^{\prime}\left(\xi_{y}\right), \\
\partial_{x} \mathcal{D}_{\text {implicit }}^{j i}(y, x) & =\left(\partial_{x} \mathcal{E}_{2}\right)(T(y, x), x)=L_{i}^{\prime}\left(\xi_{x}\right) .
\end{aligned}
$$

Writing $\tau$ for $T(y, x)$, and using the optimality condition (6.26), we get

$$
\begin{aligned}
\left(\mathcal{D}_{\text {implicit }}^{j i}-x \partial_{x} \mathcal{D}_{\text {implicit }}^{j i}-y \partial_{y} \mathcal{D}_{\text {implicit }}^{j i}\right)(y, x) & =\tau K_{j}\left(-\frac{y}{\tau}\right)+(1-\tau) K_{i}\left(\frac{x}{1-\tau}\right)+L_{0}(0) \\
& =K_{j}\left(-\frac{y}{\tau}\right)+L_{0}(0)=-H_{j}\left(L_{j}^{\prime}\left(-\frac{y}{\tau}\right)\right) \\
& =-H_{j}\left(-\partial_{y} \mathcal{D}_{\text {implicit }}^{j i}(y, x)\right) \\
& =K_{i}\left(\frac{x}{1-\tau}\right)+L_{0}(0)=-H_{i}\left(L_{i}^{\prime}\left(\frac{x}{1-\tau}\right)\right) \\
& =-H_{i}\left(\partial_{x} \mathcal{D}_{\text {implicit }}^{j i}(y, x)\right)
\end{aligned}
$$

where we have used (6.23) in the second and in the fourth line. Hence $\mathcal{D}_{\text {implicit }}^{j i}$ satisfies $(6.18)$ on $J_{j}^{*} \times J_{i}^{*}$.

Step 3: further study of $\mathcal{D}_{\text {junction. }}^{j i}$ We concluded at the end of Step 1 that for $(y, x) \in J_{j}^{*} \times J_{i}^{*}$ we have

$$
\mathcal{D}_{\text {junction }}^{j i}(y, x)=\mathcal{E}_{1}\left(\tau_{1}, y\right)+\mathcal{E}_{2}\left(\tau_{2}, x\right)
$$

with $0<\tau_{1} \leq \tau_{2}<1$. Then we can distinguish two cases.

Case 1: $\tau_{1}<\tau_{2}$. In that case this implies that

$$
\partial_{\tau_{1}} \mathcal{E}_{1}\left(\tau_{1}, y\right)=0, \quad \partial_{\tau_{2}} \mathcal{E}_{2}\left(\tau_{2}, x\right)=0
$$

which can be written as

$$
K_{j}\left(\xi_{y}\right)=0, \quad K_{i}\left(\xi_{x}\right)=0
$$

with $\xi_{y} \leq 0 \leq \xi_{x}$ defined in (6.24). 
Using Lemma 6.17, we conclude that (6.30) holds true if and only if $K_{j}(0)>0$ and $K_{i}(0)>0 ;$ i.e. $j, i \in I_{N} \backslash I_{0}$. In this case we have $\xi_{y}=\xi_{j}^{-}$and $\xi_{x}=\xi_{i}^{+}$and then

$$
\tau_{1}=-\frac{y}{\xi_{j}^{-}}, \quad \tau_{2}=1-\frac{x}{\xi_{i}^{+}}
$$

Moreover, we have in this case $\mathcal{D}_{\text {junction }}^{j i}(y, x)=\mathcal{D}_{\text {linear }}^{j i}(y, x)$.

Using Legendre-Fenchel's equality together with $K_{j}\left(\xi_{j}^{-}\right)=0$ and $K_{i}\left(\xi_{i}^{+}\right)=0$, we have

$$
\mathcal{D}_{\text {linear }}^{j i}(y, x)-y \partial_{y} \mathcal{D}_{\text {linear }}^{j i}(y, x)-x \partial_{x} \mathcal{D}_{\text {linear }}^{j i}(y, x)=L_{0}(0)
$$

and

$$
\begin{gathered}
H_{i}\left(\partial_{x} \mathcal{D}_{\text {linear }}^{j i}(y, x)\right)=H_{i}\left(L_{i}^{\prime}\left(\xi_{i}^{+}\right)\right)=\xi_{i}^{+} L_{i}^{\prime}\left(\xi_{i}^{+}\right)-L_{i}\left(\xi_{i}^{+}\right)=-L_{0}(0), \\
H_{j}\left(-\partial_{y} \mathcal{D}_{\text {linear }}^{j i}(y, x)\right)=H_{j}\left(L_{j}^{\prime}\left(\xi_{j}^{-}\right)\right)=\xi_{j}^{-} L_{j}^{\prime}\left(\xi_{j}^{-}\right)-L_{j}\left(\xi_{j}^{-}\right)=-L_{0}(0) .
\end{gathered}
$$

Hence $\mathcal{D}_{\text {linear }}^{j i}$ satisfies $(6.18)$ on $J_{j}^{*} \times J_{i}^{*}$.

Finally we deduce from (6.31) that the condition: $0<\tau_{1}<\tau_{2}<1$ is equivalent to $(y, x) \in \Delta^{j i} \cap\left(J^{*}\right)^{2}$ and then by continuity of $\mathcal{D}_{\text {junction }}^{j i}$ and $\mathcal{D}_{\text {linear }}^{j i}$, we get

$$
\mathcal{D}_{\text {junction }}^{j i}=\mathcal{D}_{\text {linear }}^{j i} \quad \text { on } \quad \Delta^{j i} .
$$

Case 2: $\tau_{1}=\tau_{2}$. If for $(y, x) \in J_{j}^{*} \times J_{i}^{*}$ we have

$$
\mathcal{D}_{\text {junction }}^{j i}(y, x)=\mathcal{E}_{1}\left(\tau_{1}, y\right)+\mathcal{E}_{2}\left(\tau_{2}, x\right)
$$

with $\tau_{1}=\tau_{2}$, then we have seen that $(y, x) \in\left(J_{j}^{*} \times J_{i}^{*}\right) \backslash \Delta^{j i}$ and $\mathcal{D}_{\text {junction }}^{j i}(y, x)=\mathcal{D}_{\text {implicit }}^{j i}(y, x)$. From Lemma 6.11, we also have that $\mathcal{D}_{\text {implicit }}^{j i} \in C\left(J_{j} \times J_{i}\right)$ if $j \in I_{0}$ or $i \in I_{0}$ and in that case $\Delta^{j i}=\emptyset$. On the other hand, we have $\mathcal{D}_{\text {implicit }}^{j i} \in C\left(\left(J_{j} \times J_{i}\right) \backslash\{(0,0)\}\right)$ if $j, i \in I_{N} \backslash I_{0}$ with $\{(0,0)\} \in \Delta^{i j}$ in that case. Therefore in all cases we have

$$
\mathcal{D}_{\text {implicit }}^{j i} \in C\left(\left(J_{j} \times J_{i}\right) \backslash \Delta^{j i}\right) .
$$

Now from the continuity of $\mathcal{D}_{\text {junction, }}$, we deduce that

$$
\mathcal{D}_{\text {junction }}^{j i}=\mathcal{D}_{\text {implicit }}^{j i} \quad \text { on } \quad\left(J_{j} \times J_{i}\right) \backslash \Delta^{j i} .
$$

Step 4: on the boundary $\left(\partial \Delta^{j i}\right) \cap\left(J^{*}\right)^{2}$. We already know that $\mathcal{D}_{\text {junction }}$ is continuous, therefore if $j, i \in I_{N} \backslash I_{0}$ :

$$
\mathcal{D}_{\text {linear }}^{j i}=\mathcal{D}_{\text {implicit }}^{j i} \quad \text { on } \quad\left\{(y, x) \in J_{j} \times J_{i}, \quad \frac{x}{\xi_{i}^{+}}-\frac{y}{\xi_{j}^{-}}=1\right\} .
$$

On the other hand, recall that $(y, x) \in J_{j}^{*} \times J_{i}^{*}$, the real $\tau \in(0,1)$ is characterized by (6.26), i.e.

$$
K_{j}\left(\xi_{y}\right)=K_{i}\left(\xi_{x}\right) \quad \text { with } \quad \xi_{y}=-\frac{y}{\tau}, \quad \xi_{x}=\frac{x}{1-\tau} .
$$

Notice that if we choose

$$
\tau=-\frac{y}{\xi_{j}^{-}}
$$

we deduce from $\frac{x}{\xi_{i}^{+}}-\frac{y}{\xi_{j}^{-}}=1$ that

$$
\xi_{y}=\xi_{j}^{-} \quad \text { and } \quad \xi_{x}=\xi_{i}^{+}
$$

which are obvious solutions of (6.33). Therefore we conclude that this is the solution. Using (6.28)-(6.29) and the expression of $\mathcal{D}_{\text {linear }}^{j i},(6.34)$ implies the equality of the gradients of $\mathcal{D}_{\text {linear }}^{j i}$ and $\mathcal{D}_{\text {implicit }}^{j i}$ on the boundary $\left(\partial \Delta^{j i}\right) \cap\left(J^{*}\right)^{2}$. Finally this shows that $\mathcal{D}_{\text {junction }}^{j i} \in C^{1}\left(J_{j}^{*} \times J_{i}^{*}\right)$. This ends the proof of the lemmas. 
Proof of Lemma 6.15. The proof proceeds in several steps.

Continuity of $T$. We set for $(\tau, y, x) \in[0,1] \times J_{j} \times J_{i}$

$$
e(\tau, y, x)=\mathcal{E}_{1}(\tau, y)+\mathcal{E}_{2}(\tau, x) .
$$

From Proposition 6.12, we already know that there exists a unique $\tau \in[0,1]$ such that

$$
\mathcal{D}_{\text {implicit }}^{j i}(y, x)=e(\tau, y, x) \quad \text { if } \quad(y, x) \in J_{j}^{*} \times J_{i}^{*} .
$$

On the other hand, we have

$$
e(\tau, y, x)= \begin{cases}(1-\tau) L_{i}\left(\frac{x}{1-\tau}\right)+\tau L_{0}(0) & \text { if }(y, x) \in\{0\} \times J_{i}^{*}(\text { case } 1), \\ \tau L_{j}\left(-\frac{y}{\tau}\right)+(1-\tau) L_{0}(0) & \text { if }(y, x) \in J_{j}^{*} \times\{0\} \text { (case 2). }\end{cases}
$$

Notice that in Cases 1 and 2, there is a unique $\tau \in[0,1]$ such that

$$
\mathcal{D}_{\text {implicit }}^{j i}(y, x)=e(\tau, y, x)
$$

and $\tau \in[0,1)$ in case $1, \tau \in(0,1]$ in case 2 . Then the continuity of $\tau=T(y, x)$ in $\left(J_{j} \times J_{i}\right) \backslash\{(0,0)\}$ follows from the lower semi-continuity of $e$ on $[0,1] \times J_{j} \times J_{i}$ and the uniqueness of $\tau$ such that (6.36) holds.

Computation of $T$. We distinguish cases.

Case 1: $(y, x) \in\left(\{0\} \times J_{i}^{*}\right) \backslash \Delta^{j i}$. Notice that we have

$$
\partial_{\tau} e(\tau, 0, x)=-K_{i}\left(\xi_{x}\right) \quad \text { with } \quad \xi_{x}=\frac{x}{1-\tau} .
$$

If $x \geq \xi_{i}^{+}$, then $\partial_{\tau} e(\tau, 0, x) \geq 0$ and $T(0, x)=0$.

If $x<\xi_{i}^{+}$, then $\xi_{x}=\xi_{i}^{+}$is a solution of $\partial_{\tau} e(\tau, 0, x)=-K_{i}\left(\xi_{x}\right)=0$ and $T(0, x)=1-\frac{x}{\xi_{i}^{+}}$.

Case 2: $(y, x) \in\left(J_{j}^{*} \times\{0\}\right) \backslash \Delta^{j i}$. Notice that we have

$$
\partial_{\tau} e(\tau, y, 0)=K_{j}\left(\xi_{y}\right) \quad \text { with } \quad \xi_{y}=-\frac{y}{\tau} .
$$

If $y \geq-\xi_{j}^{-}$, then $\partial_{\tau} e(\tau, y, 0) \leq 0$ and $T(y, 0)=1$.

If $y<-\xi_{j}^{-}$, then $\xi_{y}=\xi_{j}^{-}$is a solution of $\partial_{\tau} e(\tau, y, 0)=K_{j}\left(\xi_{y}\right)=0$ and $T(y, 0)=-\frac{y}{\xi_{j}^{-}}$. This ends the proof of the lemma.

Proof of Lemma 6.16. The proof proceeds in several steps.

Step 1: continuity. From Proposition 6.12, we already know that $\mathcal{D}_{\text {junction }}^{j i} \in C^{1}\left(\left(J_{j}^{*} \times J_{i}^{*}\right) \cup \Delta^{j i}\right)$ and $(6.19)$ holds true with

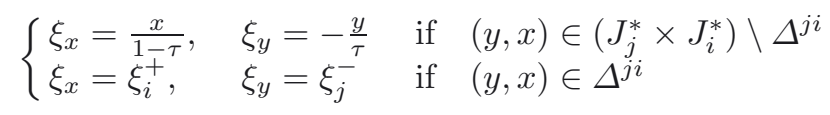

where $\tau=T(y, x)$ in the first line. Therefore, in order to prove that $\mathcal{D}_{\text {junction }}^{j i} \in C^{1}\left(J_{j} \times J_{i}\right)$, it is sufficient to prove that if $(y, x) \in\left(\partial\left(J_{j} \times J_{i}\right)\right) \backslash \Delta^{j i}=\left(\left(\{0\} \times J_{i}\right) \times\left(J_{j} \times\{0\}\right)\right) \backslash \Delta^{j i}$, and if $\left(y^{k}, x^{k}\right) \in\left(J_{j}^{*} \times J_{i}^{*}\right) \backslash \Delta^{j i}$ is a sequence of points such that $\left(y^{k}, x^{k}\right) \rightarrow(y, x)$, then we have with $\tau^{k}=T\left(y^{k}, x^{k}\right)$

$$
-\frac{y^{k}}{\tau^{k}} \rightarrow \xi_{y} \quad \text { and } \quad \frac{x^{k}}{1-\tau^{k}} \rightarrow \xi_{x}
$$


where $\left(\xi_{y}, \xi_{x}\right)$ is given by $(6.20)$. Let us recall that $\tau^{k}$ is characterized by the equation

$$
K_{j}\left(-\frac{y^{k}}{\tau^{k}}\right)=K_{i}\left(\frac{x^{k}}{1-\tau^{k}}\right)
$$

We will assume (up to extract a subsequence) that $\tau^{k} \rightarrow \tau_{0}$ for some limit $\tau_{0} \in[0,1]$. Because we have $\left|x^{k}\right|^{2}+\left|y^{k}\right|^{2} \leq R^{2}$, it is easy to deduce from (6.38), that there exists a constant $C_{R}$ such that

$$
\left|-\frac{y^{k}}{\tau^{k}}\right|+\left|\frac{x^{k}}{1-\tau^{k}}\right| \leq C_{R}
$$

This can be proved by contradiction, distinguishing the cases $\tau_{0}=0, \tau_{0}=1$ and $\tau_{0} \in(0,1)$. Up to extract a subsequence, we can then pass to the limit in (6.38) and get

$$
K_{j}\left(\xi_{y}\right)=K_{i}\left(\xi_{x}\right) \quad \text { with } \quad \xi_{y} \leq 0 \leq \xi_{x} .
$$

In the following cases, we now identify one of the two quantities $\xi_{y}$ or $\xi_{x}$, the other one being determined by (6.40).

Case 1: $(y, x) \in\left(\{0\} \times J_{i}^{*}\right) \backslash \Delta^{j i}$. From Lemma 6.15 , we know that $\tau_{0}=\max \left(0,1-\frac{x}{\xi_{i}^{+}}\right)$, and then

$$
\xi_{x}=\max \left(x, \xi_{i}^{+}\right), \quad \xi_{y}=\left(K_{j}^{-}\right)^{-1}\left(K_{i}\left(\xi_{x}\right)\right)
$$

and from (6.35), we get

$$
\mathcal{D}_{\text {junction }}^{j i}(0, x)=\frac{x}{\xi_{x}}\left(L_{i}\left(\xi_{x}\right)-L_{0}(0)\right)+L_{0}(0) .
$$

Case 2: $(y, x) \in\left(J_{j}^{*} \times\{0\}\right) \backslash \Delta^{j i}$. From Lemma 6.15 , we know that $\tau_{0}=\min \left(1,-\frac{y}{\xi_{j}^{-}}\right)$, and then

$$
-\xi_{y}=\max \left(y,-\xi_{j}^{-}\right), \quad \xi_{x}=\left(K_{i}^{+}\right)^{-1}\left(K_{j}\left(\xi_{y}\right)\right)
$$

and from (6.35), we get

$$
\mathcal{D}_{\text {junction }}^{j i}(y, 0)=-\frac{y}{\xi_{y}}\left(L_{j}\left(\xi_{y}\right)-L_{0}(0)\right)+L_{0}(0) .
$$

Case 3: $(y, x) \in\{(0,0)\} \backslash \Delta^{j i}$. This case only occurs if $j \in I_{0}$ or $i \in I_{0}$. Moreover at least one of the two quantities $-\frac{y^{k}}{\tau^{k}}$ and $\frac{x^{k}}{1-\tau^{k}}$ tends to zero.

If $\xi_{y}=0$, then $K_{i}\left(\xi_{x}\right)=K_{j}(0)$ and hence

$$
\xi_{y}=0 \quad \Longrightarrow \quad L_{i}(0) \geq L_{j}(0)=L_{0}(0) .
$$

If $\xi_{x}=0$, then $K_{j}\left(\xi_{y}\right)=K_{i}(0)$ and hence

$$
\xi_{x}=0 \quad \Longrightarrow \quad L_{j}(0) \geq L_{i}(0)=L_{0}(0) .
$$

This implies that

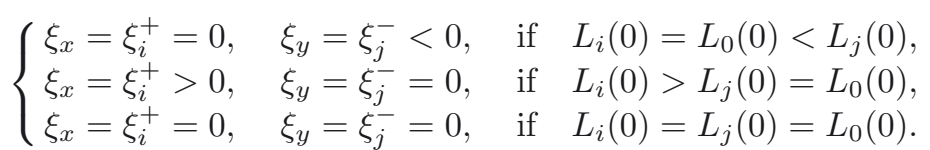

By the uniqueness of the limit, this finally shows that $\mathcal{D}_{\text {junction }}^{j i} \in C^{1}\left(J_{j} \times J_{i}\right)$ and (6.20) holds.

Step 2: checking (6.35) and (6.22). From (6.41) and (6.42), we deduce (6.35) on $\left(\left(J_{j}^{*} \times\{0\}\right) \cup\left(\{0\} \times J_{i}^{*}\right)\right) \backslash \Delta^{j i}$. From $\mathcal{D}_{\text {junction }}^{j i}=\mathcal{D}_{\text {linear }}^{j i}$ on $\Delta^{j i}$, we deduce that $(6.35)$ is also true on $\left(\left(J_{j}^{*} \times\{0\}\right) \cup\left(\{0\} \times J_{i}^{*}\right)\right) \cap \Delta^{j i}$.

Then (6.22) follows from a simple computation for $(y, x) \neq(0,0)$. This is still true for $(y, x)=0$, because $\mathcal{D}_{\text {junction }}^{j i}$ is $C^{1}$. This ends the proof of the lemma. 


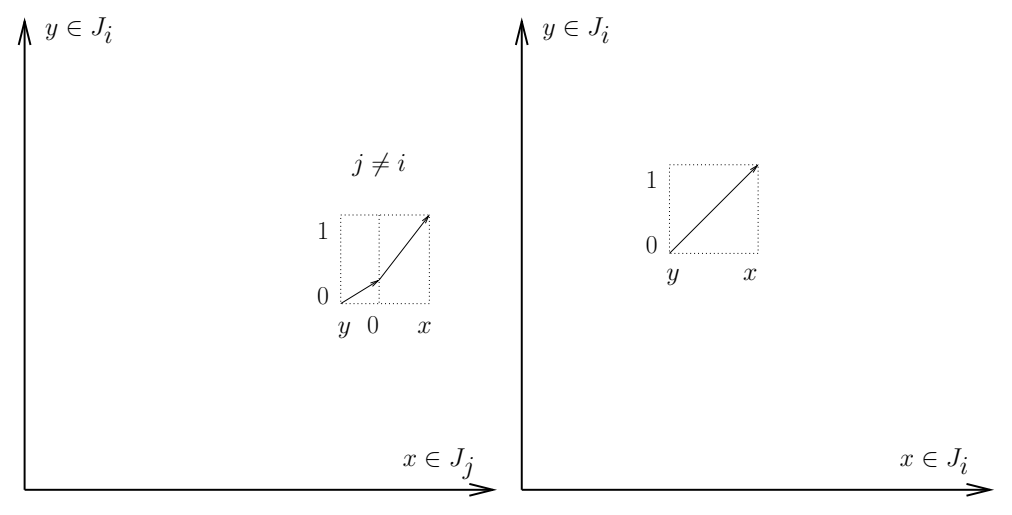

Figure 3. $i \in I_{0}$ or $j \in I_{0}$ : trajectories never stay at the junction point.

\subsection{Study of $\mathcal{D}_{\text {straight }}$}

The following lemma will be used below. Since it is elementary, its proof is omitted.

Lemma 6.18 (properties of $\mathcal{D}_{\text {straight }}^{j i}$ ). For $j=i \in I_{N}$, we have for $(y, x) \in J_{j} \times J_{i}$ with $(y, x) \neq(0,0)$ if $j=i \in I_{N} \backslash I_{0}$ :

$$
\begin{aligned}
\mathcal{D}_{\text {straight }}^{j i}(y, x)-x \partial_{x} \mathcal{D}_{\text {straight }}^{j i}(y, x)-y \partial_{y} \mathcal{D}_{\text {straight }}^{j i}(y, x) & =L_{0}(0)+K_{i}(x-y) \\
& =-H_{i}\left(\partial_{x} \mathcal{D}_{\text {straight }}^{j i}(y, x)\right)-H_{j}\left(-\partial_{y} \mathcal{D}_{\text {straight }}^{j i}(y, x)\right)
\end{aligned}
$$

and

$$
\partial_{x} \mathcal{D}_{\text {straight }}^{j i}(y, x)=L_{i}^{\prime}(x-y), \quad \partial_{y} \mathcal{D}_{\text {straight }}^{j i}(y, x)=-L_{j}^{\prime}(x-y) .
$$

\subsection{Proof of Theorem 6.2}

We are now in position to prove Theorem 6.2. We prove several lemmas successively.

Lemma 6.19 (properties of $\left.\mathcal{D}_{0}^{j i}\right)$. For $(y, x) \in J_{j} \times J_{i}$, we have

$$
\mathcal{D}_{0}^{j i}(y, x)= \begin{cases}L_{i}(x-y) & \text { if } i=j \in I_{0}, \\ \mathcal{D}_{\text {junction }}^{j i}(y, x) & \text { if } i \neq j, \\ \min \left(\mathcal{D}_{\text {junction }}^{j i}(y, x), L_{i}(x-y)\right) & \text { if } i=j \in I_{N} \backslash I_{0} .\end{cases}
$$

In particular $\mathcal{D}_{0}^{j i} \in C^{1}\left(J_{j} \times J_{i}\right)$ in the first two cases.

Lemma 6.20 (singularities of the gradient of $\mathcal{D}_{0}$ ). In the case $i=j \in I_{N} \backslash I_{0}$, we have

$$
\mathcal{D}_{0}^{j i}(y, x)= \begin{cases}\mathcal{D}_{\text {linear }}^{j i} & \text { in a neighborhood of }\left(\partial\left(J_{j} \times J_{i}\right)\right) \cap \overline{\Delta^{j i}} \\ L_{i}(x-y) & \text { in a neighborhood of }\left(\partial\left(J_{j} \times J_{i}\right)\right) \backslash \overline{\Delta^{j i}}\end{cases}
$$

moreover, in this case there exists a curve $\Gamma^{j i}$ such that $\mathcal{D}_{0}^{j i} \in C^{1}\left(\left(J_{j} \times J_{i}\right) \backslash\left(\Gamma^{j i} \cup\left\{Y_{j}, X_{i}\right\}\right)\right)$. This curve connects $Y_{j}=\left(-\xi_{j}^{-}, 0\right)$ and $X_{i}=\left(0, \xi_{i}^{+}\right)$and is contained in $\left(J_{j}^{*} \times J_{i}^{*}\right) \cap \Delta^{j i}$

The results of these two lemmas are illustrated in Figures 3 and 4. 

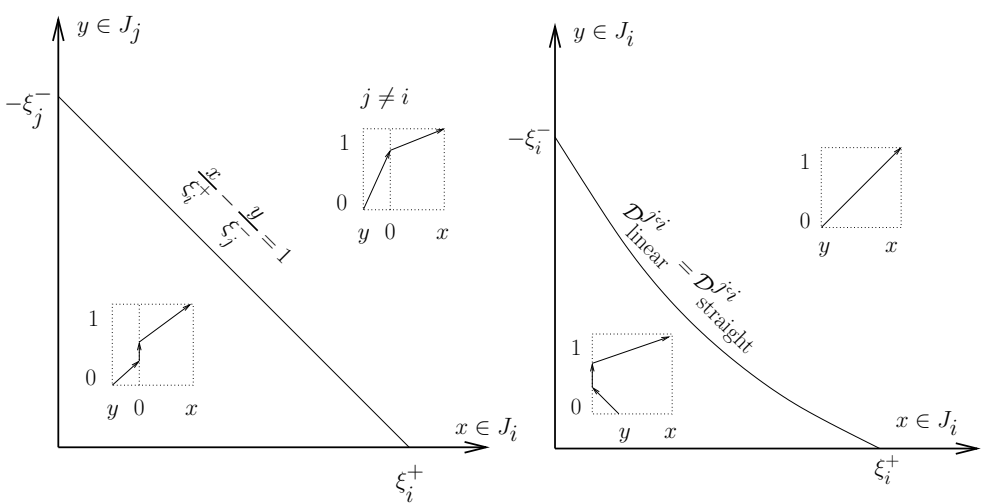

Figure 4. $i, j \in I_{N} \backslash I_{0}$ : trajectories do stay at the junction point if $(y, x) \sim(0,0)$.

Proof of Lemma 6.19. We only have to treat the case $i=j$. The convexity of $L_{i}$ implies that for $\tau \in(0,1)$ :

$$
e(\tau, y, x)=\tau L_{i}\left(-\frac{y}{\tau}\right)+(1-\tau) L_{i}\left(\frac{x}{1-\tau}\right) \geq L_{i}(x-y) .
$$

Therefore for $(y, x) \in J_{j}^{*} \times J_{i}^{*}$ with $j=i$, we have

$$
\mathcal{D}_{\text {implicit }}^{j i}(y, x)=\inf _{0<\tau<1} e(\tau, y, x) \geq L_{i}(x-y) .
$$

When $i=j \in I_{0}$, we have $\mathcal{D}_{\text {junction }}^{j i}(y, x)=\mathcal{D}_{\text {implicit }}^{j i}(y, x)$, and then

$$
\mathcal{D}_{\text {junction }}^{j i}(y, x) \geq L_{i}(x-y)=\mathcal{D}_{\text {straight }}^{j i}(y, x)=\mathcal{D}_{0}^{j i}(y, x)
$$

for $(y, x) \in J_{j}^{*} \times J_{i}^{*}$ and then also for $(y, x) \in J_{j} \times J_{i}$, by continuity of the functions.

Proof of Lemma 6.20. We first prove (6.43) and then describe the curve $\Gamma_{j, i}$.

Proof of (6.43). Combining (6.13) and (6.17), we obtain

$$
\begin{aligned}
\mathcal{D}_{0}^{j i}(y, x) & =\min \left(\mathcal{D}_{\text {junction }}^{j i}(y, x), \mathcal{D}_{\text {straight }}^{j i}(y, x)\right) \\
& = \begin{cases}\mathcal{D}_{\text {straight }}^{j i}(y, x)=L_{i}(x-y) & \text { for }(y, x) \in\left(J_{j} \times J_{i}\right) \backslash \Delta^{j i}, \\
\min \left(\mathcal{D}_{\text {linear }}^{j i}(y, x), \mathcal{D}_{\text {straight }}^{j i}(y, x)\right) & \text { for }(y, x) \in \Delta^{j i}\end{cases}
\end{aligned}
$$

On the other hand, we have (a strictly convex function being above its tangent) for $x \neq \xi_{i}^{+}$and $y \neq-\xi_{j}^{-}$

$$
\begin{gathered}
L_{i}(x)>L_{i}\left(\xi_{i}^{+}\right)+\left(x-\xi_{i}^{+}\right) L_{i}^{\prime}\left(\xi_{i}^{+}\right)=x L_{i}^{\prime}\left(\xi_{i}^{+}\right)+L_{0}(0)=\mathcal{D}_{\text {linear }}^{j i}(0, x) \\
L_{j}(-y)>L_{j}\left(\xi_{j}^{-}\right)+\left(-y-\xi_{j}^{-}\right) L_{j}^{\prime}\left(\xi_{j}^{-}\right)=-y L_{j}^{\prime}\left(\xi_{j}^{-}\right)+L_{0}(0)=\mathcal{D}_{\text {linear }}^{j i}(y, 0) .
\end{gathered}
$$

This shows that

$$
\mathcal{D}_{\text {straight }}^{j i}>\mathcal{D}_{\text {linear }}^{j i} \quad \text { on } \quad\left(\partial\left(J_{j} \times J_{i}\right)\right) \cap \Delta^{j i} .
$$

We see that (6.44) and (6.45) imply (6.43). 
Description of $\left\{\mathcal{D}_{\text {linear }}^{j i}=\mathcal{D}_{\text {straight }}^{j i}\right\} \cap \Delta^{j i}$. Notice that

$$
\left\{\begin{array}{l}
\mathcal{D}_{\text {linear }}^{j i}\left(0, \xi_{i}^{+}\right)=\xi_{i}^{+} L_{i}^{\prime}\left(\xi_{i}^{+}\right)+L_{0}(0)=L_{i}\left(\xi_{i}^{+}\right)=\mathcal{D}_{\text {straight }}^{j i}\left(0, \xi_{i}^{+}\right), \\
\mathcal{D}_{\text {linear }}^{j i}\left(-\xi_{j}^{-}, 0\right)=\xi_{j}^{-} L_{j}^{\prime}\left(\xi_{j}^{-}\right)+L_{0}(0)=L_{j}\left(\xi_{j}^{-}\right)=\mathcal{D}_{\text {straight }}^{j i}\left(-\xi_{j}^{-}, 0\right) .
\end{array}\right.
$$

This means that the functions $\mathcal{D}_{\text {linear }}^{j i}$ and $\mathcal{D}_{\text {straight }}^{j i}$ coincide at the two points $X_{i}=\left(0, \xi_{i}^{+}\right)$and $Y_{j}=\left(-\xi_{j}^{-}, 0\right)$. Therefore we have

$$
\left.\mathcal{D}_{\text {straight }}^{j i}<\mathcal{D}_{\text {linear }}^{j i} \quad \text { on the open interval }\right] X_{i}, Y_{j}[
$$

because $\mathcal{D}_{\text {linear }}^{j i}$ is linear and $\mathcal{D}_{\text {straight }}^{j i}$ is strictly convex as a function of $y-x$.

The function $(y, x) \mapsto \mathcal{D}_{\text {straight }}^{j i}(y, x)-\mathcal{D}_{\text {linear }}^{j i}(y, x)$ being convex because $\mathcal{D}_{\text {linear }}^{j i}(y, x)$ is linear, we can consider the convex set

$$
K^{j i}=\left\{(y, x) \in J_{j} \times J_{i}, \quad \mathcal{D}_{\text {straight }}^{j i}(y, x) \leq \mathcal{D}_{\text {linear }}^{j i}(y, x)\right\} .
$$

Then for $i=j \in I_{N} \backslash I_{0}$, the set

$$
\Gamma^{j i}=\left\{(y, x) \in \Delta^{j i}, \quad \mathcal{D}_{\text {linear }}^{j i}(y, x)=\mathcal{D}_{\text {straight }}^{j i}(y, x)\right\}
$$

is contained in the boundary of the convex set $K^{j i}$. More precisely, we have

$$
\Gamma^{j i}=\left(\left(\partial K^{j i}\right) \cap \Delta^{j i}\right) \subset J_{j}^{*} \times J_{i}^{*}
$$

which shows that $\Gamma^{j i}$ is a curve and

$$
\overline{\Gamma^{j i}}=\Gamma^{j i} \cup\left\{X_{i}, Y_{j}\right\}
$$

Lemma 6.21 (the equations for $\mathcal{D}_{0}$ ). For all $i, j$, and $x, y$ where $\mathcal{D}_{0}^{j i}$ is $C^{1}$ :

$$
\left(\mathcal{D}_{0}^{j i}-x \partial_{x} \mathcal{D}_{0}^{j i}-y \partial_{y} \mathcal{D}_{0}^{j i}\right)(y, x)=-H_{i}\left(\left(\partial_{x} \mathcal{D}_{0}^{j i}\right)(y, x)\right)=-H_{j}\left(\left(-\partial_{y} \mathcal{D}_{0}^{j i}\right)(y, x)\right) .
$$

Moreover for all $x \in J_{i}$ (with $x \neq \xi_{i}^{+}$if $j=i \in I_{N} \backslash I_{0}$ )

$$
\left(\mathcal{D}_{0}^{j i}-x \partial_{x} \mathcal{D}_{0}^{j i}-y \partial_{y} \mathcal{D}_{0}^{j i}\right)(0, x)=L_{0}(0)+K_{i}\left(\max \left(x, \xi_{i}^{+}\right)\right)
$$

and for all $y \in J_{j}$ (with $y \neq-\xi_{j}^{-}$if $j=i \in I_{N} \backslash I_{0}$ )

$$
\left(\mathcal{D}_{0}^{j i}-x \partial_{x} \mathcal{D}_{0}^{j i}-y \partial_{y} \mathcal{D}_{0}^{j i}\right)(y, 0)=L_{0}(0)+K_{j}\left(-\max \left(y,-\xi_{j}^{-}\right)\right) .
$$

We also have

$$
\partial_{x} \mathcal{D}_{0}^{j i}(y, x)=L_{i}^{\prime}\left(\xi_{x}\right), \quad \partial_{y} \mathcal{D}_{0}^{j i}(y, x)=-L_{j}^{\prime}\left(\xi_{y}\right)
$$

for all $(y, x) \in \partial\left(J_{j} \times J_{i}\right)$ except for $i=j \in I_{N} \backslash I_{0}$ for which we exclude points $(y, x) \in\left\{Y_{j}, X_{i}\right\}$.

Moreover for $j=i \in I_{0}$, we have

$$
\xi_{x}=\xi_{y}=x-y \quad \text { for all } \quad(y, x) \in \partial\left(J_{j} \times J_{i}\right)
$$

and $j=i \in I_{N} \backslash I_{0}$, we have

$$
\left\{\begin{array}{ll}
\xi_{y}=x-y, & \xi_{x}=x-y \\
\xi_{y}=\xi_{j}^{-}, & \xi_{x}=\xi_{i}^{+}
\end{array} \quad \text { for } \quad(y, x) \in\left(\partial\left(J_{j} \times J_{i}\right)\right) \cap \overline{\Delta^{j i}} .\right.
$$


Proof. Using Proposition 6.12 for $\mathcal{D}_{\text {junction }}^{j i}$, Lemma 6.18 for $\mathcal{D}_{\text {straight }}^{j i}$, and (6.32) for $\mathcal{D}_{\text {linear }}^{j i}$ and the property (6.43), we get

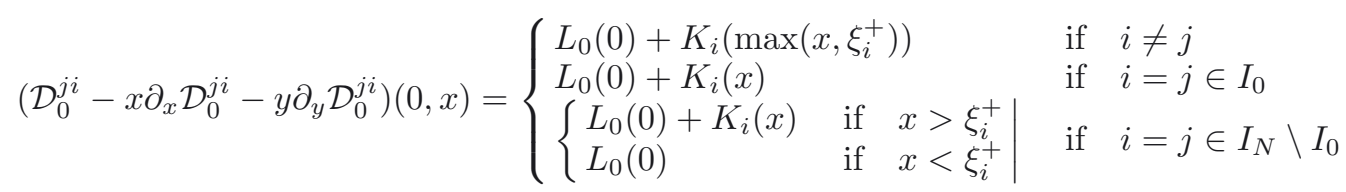

which implies (6.47). Similarly we get

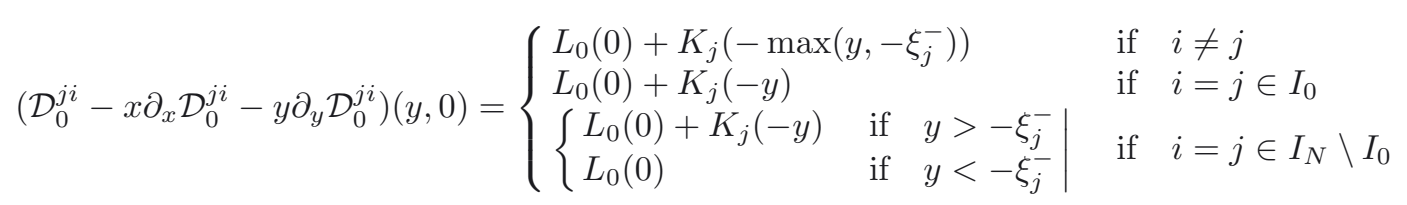

which implies (6.48). Relations (6.46) and (6.49) follow both from Proposition 6.12 and Lemma 6.18. Finally (6.50) and (6.51) follows from the previous results. This ends the proof of the lemma.

We now can check the equations satisfied by $\mathcal{D}_{0}$ at the boundary.

Lemma 6.22 (boundary properties of $\left.\mathcal{D}_{0}\right)$. At any point $(y, x) \in\{0\} \times J_{i}$ with $x \neq \xi_{i}^{+}$if $i \in I_{N} \backslash I_{0}$, we have for any $j \in I_{N}$

$$
\left(\mathcal{D}_{0}^{j i}-x \partial_{x} \mathcal{D}_{0}^{j i}-y \partial_{y} \mathcal{D}_{0}^{j i}\right)(y, x)= \begin{cases}-\max _{k \in I_{N}} H_{k}^{-}\left(-\partial_{y} \mathcal{D}_{0}^{k i}(y, x)\right) & \text { if } \quad N \geq 2, \\ -H_{1}\left(-\partial_{y} \mathcal{D}_{0}^{k i}(y, x)\right) & \text { if } \quad N=1 .\end{cases}
$$

Lemma 6.23 (boundary properties of $\mathcal{D}_{0}$ (continued)). At any point $(y, x) \in J_{j} \times\{0\}$ with $y \neq-\xi_{j}^{-}$if $j \in I_{N} \backslash I_{0}$, we have for any $i \in I_{N}$

$$
\left(\mathcal{D}_{0}^{j i}-x \partial_{x} \mathcal{D}_{0}^{j i}-y \partial_{y} \mathcal{D}_{0}^{j i}\right)(y, x)=-\max _{k \in I_{N}} H_{k}^{-}\left(\partial_{x} \mathcal{D}_{0}^{j k}(y, x)\right) .
$$

Proof of Lemma 6.22. We first remark the general fact that

$$
H_{k}\left(L_{k}^{\prime}(\xi)\right)=H_{k}^{-}\left(L_{k}^{\prime}(\xi)\right) \text { if } \quad \xi \leq 0 .
$$

On the one hand, from Lemma 6.19, we have for points $(y, x) \in\{0\} \times J_{i}$ where $\mathcal{D}_{0}^{k i}$ is $C^{1}$

$$
-\left(\mathcal{D}_{0}^{k i}-x \partial_{x} \mathcal{D}_{0}^{k i}-y \partial_{y} \mathcal{D}_{0}^{k i}\right)(y, x)=H_{k}\left(-\partial_{y} \mathcal{D}_{0}^{k i}(y, x)\right) \geq H_{k}^{-}\left(-\partial_{y} \mathcal{D}_{0}^{k i}(y, x)\right)
$$

and this common quantity is independent on $k$. Therefore to conclude to (6.52) in the case $N \geq 2$, it is enough to show that there exists at least an index $k$ such that

$$
H_{k}\left(-\partial_{y} \mathcal{D}_{0}^{k i}(y, x)\right)=H_{k}^{-}\left(-\partial_{y} \mathcal{D}_{0}^{k i}(y, x)\right)
$$

Case 1: $N \geq 2$ and $k \neq i$. Then we have $\xi_{y} \leq 0$ and then

$$
H_{k}\left(-\partial_{y} \mathcal{D}_{0}^{k i}(y, x)\right)=H_{k}\left(L_{k}^{\prime}\left(\xi_{y}\right)\right)=H_{k}^{-}\left(L_{k}^{\prime}\left(\xi_{y}\right)\right)=H_{k}^{-}\left(-\partial_{y} \mathcal{D}_{0}^{k i}(y, x)\right) .
$$

Therefore (6.54) holds true for $k \neq i$. 
Case 2: $N=1$ and $k=i=1 \in I_{0}$. Then we have

$$
\mathcal{D}_{0}(y, x)=\mathcal{D}_{0}^{11}(y, x)=L_{1}(x-y)
$$

and by Lemma 6.18 , we have for

$$
\left(\mathcal{D}_{0}^{11}-x \partial_{x} \mathcal{D}_{0}^{11}-y \partial_{y} \mathcal{D}_{0}^{11}\right)(y, x)=-H_{1}\left(-\partial_{y} \mathcal{D}_{0}^{11}(y, x)\right)
$$

which is in particular true for $y=0$. This shows (6.52) in the case $N=1$.

Proof of Lemma 6.23. From Lemma 6.19, we have for points $(y, x) \in J_{j} \times\{0\}$ where $\mathcal{D}_{0}^{j k}$ is $C^{1}$

$$
-\left(\mathcal{D}_{0}^{j k}-x \partial_{x} \mathcal{D}_{0}^{j k}-y \partial_{y} \mathcal{D}_{0}^{j k}\right)(y, x)=H_{k}\left(\partial_{x} \mathcal{D}_{0}^{j k}(y, x)\right) \geq H_{k}^{-}\left(\partial_{x} \mathcal{D}_{0}^{j k}(y, x)\right)
$$

and this common quantity is independent on $k$. Therefore to conclude to (6.53), it is enough to show that there exists at least an index $k$ such that

$$
H_{k}\left(\partial_{x} \mathcal{D}_{0}^{j k}(y, x)\right)=H_{k}^{-}\left(\partial_{x} \mathcal{D}_{0}^{j k}(y, x)\right) .
$$

Case 1: $j \in I_{0}$. Then from Lemma 6.19, we have with $k=j$

$$
\partial_{x} \mathcal{D}_{0}^{j k}(y, x)=L_{k}^{\prime}\left(\xi_{x}\right) \quad \text { with } \quad \xi_{x}=x-y \leq 0 .
$$

Therefore (6.53) holds true for $k=j$.

Case 2: $j \in I_{N} \backslash I_{0}$. We distinguish sub-cases.

Subcase 2.1: $y>-\xi_{j}^{-}$. From Lemma 6.19, we still have (6.56) with $k=j$, which again implies (6.53) for $k=j$.

Subcase 2.2: $y<-\xi_{j}^{-}$. Then we choose an index $k \in I_{0}$, and Lemma 6.19 implies that

$$
\partial_{x} \mathcal{D}_{0}^{j k}(y, x)=L_{k}^{\prime}\left(\xi_{x}\right) \quad \text { with } \quad \xi_{x}=\xi_{k}^{+}=0
$$

which again implies (6.53) for such $k \in I_{0}$. This ends the proof of the lemma.

We can now prove Theorem 6.2.

Proof of Theorem 6.2. From Lemma 6.19, we know that $\mathcal{D}_{0}$ has the regularity $C_{*}^{1}$ except on certain curves $\Gamma^{j i} \cup\left\{Y_{j}, X_{i}\right\}$ for $j=i \in I_{N} \backslash I_{0}$. So if $(y, x)$ is a point of local $C_{*}^{1}$ regularity of $\mathcal{D}_{0}$, then we simply set

$$
\varphi_{0}=\mathcal{D}_{0} \quad \text { locally around }(y, x) .
$$

If $(y, x)$ is a point where $\mathcal{D}_{0}$ is not $C_{*}^{1}$, then we have $\mathcal{D}_{0}(y, x)=\mathcal{D}_{\text {junction }}(y, x)$, and we can simply set

$$
\varphi_{0}=\mathcal{D}_{\text {junction }} \quad \text { on } J^{2} .
$$

The required equalities follow from Lemmas 6.19, 6.22 and 6.23. Estimate (6.6) follows from the fact that $\mathcal{D}_{0}$ is the minimum of $\mathcal{D}_{\text {junction }} \in C_{*}^{1}\left(J^{2}\right)$ and of functions in $C^{1}\left(J_{i}^{2}\right)$ for some $i$. This ends the proof of the theorem.

\section{Appendix A. Stability And Perron's Method}

This section contains classical results from viscosity solutions, whose statements are adapted to the equation studied in the present paper. 


\section{A.1. Stability results}

In view of Proposition 3.3, the following stability results are classical in the viscosity solution framework. See for instance [7].

Proposition A.1 (stability). Assume (A1') and let $T>0$.

- Consider a family of sub-solutions (resp. super-solutions) $\left(u_{\alpha}\right)_{\alpha \in A}$ of (1.1) on $J_{T}$ such that the u.s.c. (resp. l.s.c.) envelope $u$ of

$$
\sup _{\alpha \in A} u_{\alpha} \quad\left(\text { resp. } \inf _{\alpha \in A} u_{\alpha}\right)
$$

is finite everywhere. Then $u$ is a sub-solution (resp. super-solution) of (1.1) on $J_{T}$;

- consider a family of sub-solutions (resp. super-solutions) $\left(u_{\varepsilon}\right)_{\varepsilon \in(0,1)}$ of (1.1) on $J_{T}$ such that the upper (resp. lower) relaxed semi-limit $u$ is finite everywhere. Then $u$ is a sub-solution (resp. super-solution) of (1.1) on $J_{T}$.

\section{A.2. Perron's method}

In this subsection, we state the existence of a solution of (1.1)-(1.2) which can be constructed by using Perron's method. This method is the classical way to get existence in a viscosity solution framework.

Theorem A.2 (existence). Assume (A0)-(A1') and let $T>0$. Then there exists an upper semi-continuous function $u:[0, T) \times J \rightarrow \mathbb{R}$ which is a viscosity solution of (1.1)-(1.2) on $J_{T}$ and satisfies

$$
\left|u(t, x)-u_{0}(x)\right| \leq C t \quad \text { for } \quad(t, x) \in[0, T) \times J .
$$

Acknowledgements. The first author is partially supported by the KAMFAIBLE project (ANR-07-BLAN-0361). The authors thank A. Briani for useful discussions and important indications on the recent literature on the subject. The authors are very grateful to J.-P. Lebacque for fruitful and enlightening discussions on the modeling of traffic flows and indications on the related literature. The second author thanks M. Khoshyaran for discussions on the topic. The authors also thank Y. Achdou, G. Barles, P. Cardaliaguet, E. Chasseigne, H. Frankowska and M. Garavello for discussions about this work.

\section{REFERENCES}

[1] Y. Achdou, F. Camilli, A. Cutri and N. Tchou, Hamilton-Jacobi equations on networks. Tech. Rep., preprint HAL 00503910 (2010).

[2] Y. Achdou, F. Camilli, A. Cutri and N. Tchou, Hamilton-Jacobi equations on networks, in 18th IFAC World Congress. Milano, Italy (2011).

[3] F. Bachmann and J. Vovelle, Existence and uniqueness of entropy solution of scalar conservation laws with a flux function involving discontinuous coefficients. Comm. Partial Differential Equations 31 (2006) 371-395.

[4] M. Bardi and I. Capuzzo-Dolcetta, Optimal control and viscosity solutions of Hamilton-Jacobi-Bellman equations. Systems \& Control: Foundations \& Applications, Birkhäuser Boston Inc., Boston, MA (1997). With appendices by Maurizio Falcone and Pierpaolo Soravia.

[5] C. Bardos, A.Y. le Roux and J.-C. Nédélec, First order quasilinear equations with boundary conditions. Comm. Partial Differential Equations 4 (1979) 1017-1034.

[6] G. Barles, Discontinuous viscosity solutions of first-order Hamilton-Jacobi equations: a guided visit. Nonlinear Anal. 20 (1993) 1123-1134.

[7] G. Barles, Solutions de viscosité des équations de Hamilton-Jacobi, Mathématiques \& Applications (Berlin) [Mathematics EG Applications] 17, Springer-Verlag, Paris (1994).

[8] A. Bressan and Y. Hong, Optimal control problems on stratified domains. Netw. Heterog. Media 2 (2007) 313-331 (electronic).

[9] A. Briani and A. Davini, Monge solutions for discontinuous Hamiltonians. ESAIM: COCV 11 (2005) 229-251 (electronic).

[10] R. Bürger and K.H. Karlsen, Conservation laws with discontinuous flux: a short introduction. J. Engrg. Math. 60 (2008) $241-247$.

[11] F. Camilli and D. Schieborn, Viscosity solutions of eikonal equations on topological networks. Preprint.

[12] X. Chen and B. Hu, Viscosity solutions of discontinuous Hamilton-Jacobi equations. Interfaces and Free Boundaries 10 (2008) $339-359$. 
[13] G.M. Coclite and N.H. Risebro, Viscosity solutions of Hamilton-Jacobi equations with discontinuous coefficients. J. Hyperbolic Differ. Equ. 4 (2007) 771-795.

[14] G. Dal Maso and H. Frankowska, Value function for Bolza problem with discontinuous Lagrangian and Hamilton-Jacobi inequalities. ESAIM: COCV $\mathbf{5}$ (2000) 369-394.

[15] K.-J. Engel, M. Kramar Fijavž, R. Nagel and E. Sikolya, Vertex control of flows in networks. Netw. Heterog. Media 3 (2008) $709-722$.

[16] A. Fathi, Théorème KAM faible et théorie de Mather sur les systèmes Lagrangiens. C. R. Acad. Sci. Paris, Sér. I Math. 324 (1997) 1043-1046.

[17] M. Garavello and B. Piccoli, Traffic flow on networks, AIMS Series on Applied Mathematics 1. American Institute of Mathematical Sciences (AIMS), Springfield, MO (2006). Conservation laws models.

[18] M. Garavello and B. Piccoli, Conservation laws on complex networks. Ann. Inst. Henri. Poincaré, Anal. Non Linéaire 26 (2009) 1925-1951.

[19] M. Garavello and P. Soravia, Optimality principles and uniqueness for Bellman equations of unbounded control problems with discontinuous running cost. Nonlinear Differential Equations Appl. 11 (2004) 271-298.

[20] M. Garavello and P. Soravia, Representation formulas for solutions of the HJI equations with discontinuous coefficients and existence of value in differential games. J. Optim. Theory Appl. 130 (2006) 209-229.

[21] M. Garavello, R. Natalini, B. Piccoli and A. Terracina, Conservation laws with discontinuous flux. Netw. Heterog. Media 2 (2007) 159-179.

[22] J.-P. Lebacque, The Godunov scheme and what it means for first order traffic flow models. Internaional Symposium on Transportation and Traffic Theory 13 (1996) 647-677.

[23] J.-P. Lebacque and M.M. Khoshyaran, Modelling vehicular traffic flow on networks using macroscopic models, in Finite volumes for complex applications II. Hermes Sci. Publ., Paris (1999) 551-558.

[24] J.-P. Lebacque and M.M. Khoshyaran, First order macroscopic traffic flow models: intersection modeling, network modeling, in Transportation and Traffic Theory, Flow, Dynamics and Human Interaction. Elsevier (2005) 365-386.

[25] M.J. Lighthill and G.B. Whitham, On kinematic waves II. A theory of traffic flow on long crowded roads. Proc. Roy. Soc. A 229 (1955) 317-145.

[26] P.-L. Lions, Generalized solutions of Hamilton-Jacobi equations, Research Notes in Math. 69. Pitman Advanced Publishing Program, Mass Boston (1982).

[27] P.-L. Lions and P.E. Souganidis, Differential games, optimal control and directional derivatives of viscosity solutions of Bellman' and Isaacs' equations. SIAM J. Control Optim. 23 (1985) 566-583.

[28] R.T. Newcomb II and J. Su, Eikonal equations with discontinuities. Differential Integral Equations 8 (1995) $1947-1960$.

[29] D.N. Ostrov, Solutions of Hamilton-Jacobi equations and scalar conservation laws with discontinuous space-time dependence. J. Differential Equations 182 (2002) 51-77.

[30] P.I. Richards, Shock waves on the highway. Operation Research 4 (1956) 42-51.

[31] D. Schieborn, Viscosity Solutions of Hamilton-Jacobi Equations of Eikonal Type on Ramified Spaces. Ph.D. thesis, EberhardKarls-Universitat Tubingen (2006).

[32] N. Seguin and J. Vovelle, Analysis and approximation of a scalar conservation law with a flux function with discontinuous coefficients. Math. Models Methods Appl. Sci. 13 (2003) 221-257.

[33] A. Siconolfi, Metric character of Hamilton-Jacobi equations. Trans. Am. Math. Soc. 355 (2003) 1987-2009 (electronic).

[34] P. Soravia, Discontinuous viscosity solutions to Dirichlet problems for Hamilton-Jacobi equations with convex Hamiltonians. Comm. Partial Differential Equations 18 (1993) 1493-1514.

[35] P. Soravia, Optimality principles and representation formulas for viscosity solutions of Hamilton-Jacobi equations. I. Equations of unbounded and degenerate control problems without uniqueness. Adv. Differential Equations 4 (1999) $275-296$.

[36] P. Soravia, Optimality principles and representation formulas for viscosity solutions of Hamilton-Jacobi equations. II. Equations of control problems with state constraints. Differential Integral Equations 12 (1999) 275-293.

[37] P. Soravia, Boundary value problems for Hamilton-Jacobi equations with discontinuous Lagrangian. Indiana Univ. Math. J. 51 (2002) 451-477.

[38] P. Soravia, Uniqueness results for fully nonlinear degenerate elliptic equations with discontinuous coefficients. Commun. Pure Appl. Anal. 5 (2006) 213-240.

[39] T. Strömberg, On viscosity solutions of irregular Hamilton-Jacobi equations. Arch. Math. (Basel) 81 (2003) 678-688. 